\title{
ENUMERATING CLASSES AND CHARACTERS OF $p$-GROUPS
}

\author{
E. A. O'BRIEN AND C. VOLL
}

\begin{abstract}
We develop general formulae for the numbers of conjugacy classes and irreducible complex characters of finite $p$-groups of nilpotency class less than $p$. This allows us to unify and generalize a number of existing enumerative results, and to obtain new such results for generalizations of relatively free $p$ groups of exponent $p$. Our main tools are the Lazard correspondence and the Kirillov orbit method.
\end{abstract}

\section{INTRODUCTION}

The study of the conjugacy classes and irreducible complex characters of groups is an active area of research. The enumeration of classes and characters of finite groups of Lie type, for instance, has played an important role in the work of Liebeck, Shalev and others; see, for instance, 25]. Motivated by a conjecture of Higman [13], the classes and characters of upper-unitriangular groups have been extensively studied; see, for example, [18, 30].

'Representation growth of groups' is an umbrella term for the asymptotic and arithmetic properties of group representations as a function of their dimensions. A key tool in the study of representation growth is the Kirillov orbit method. Where applicable, it provides a parameterization of the irreducible complex representations of a group in terms of co-adjoint orbits. It was pioneered by Kirillov in the realm of nilpotent Lie groups and later adapted to other classes of groups, including $p$-adic analytic groups, finitely generated nilpotent groups, and finite $p$-groups; see 10 , 14, 15, 20. Under certain conditions the linearization achieved by this method facilitates a description of the numbers of characters of a group in terms of geometric data attached to the dual of a Lie algebra associated with the group, such as the numbers of rational points of certain algebraic subvarieties.

Let $p$ be a prime. In this paper we employ the Kirillov orbit method to study the classes and characters of finite $p$-groups of nilpotency class less than $p$. Let $G$ be a finite $p$-group. For $i \geq 0$, we define

$$
\begin{aligned}
\operatorname{cc}_{i}(G) & =\#\left\{\text { conjugacy classes of } G \text { of cardinality } p^{i}\right\} \text { and } \\
\operatorname{ch}_{i}(G) & =\#\left\{\text { irreducible complex characters of } G \text { of degree } p^{i}\right\} .
\end{aligned}
$$

The vectors $\operatorname{cc}(G)=\left(\operatorname{cc}_{i}(G)\right)_{i}$ and $\operatorname{ch}(G)=\left(\operatorname{ch}_{i}(G)\right)_{i}$ are the class vector and the character vector of $G$, respectively. We denote by $\operatorname{cs}(G)=\left\{p^{i}: \operatorname{cc}_{i}(G) \neq 0\right\}$ the class sizes of $G$ and by $\operatorname{cd}(G)=\left\{p^{i}: \operatorname{ch}_{i}(G) \neq 0\right\}$ the character degrees of $G$. We

Received by the editors November 28, 2012 and, in revised form, August 7, 2013.

2010 Mathematics Subject Classification. Primary 20C15, 20 D15.

Key words and phrases. Finite $p$-groups, character degrees, conjugacy class sizes, Kirillov orbit methods, Lazard correspondence, relatively free $p$-groups. 
write

$$
k(G)=\sum_{i} \operatorname{cc}_{i}(G)=\sum_{i} \operatorname{ch}_{i}(G)
$$

for the class number of $G$.

Let $c$ be the nilpotency class of $G$, and assume that $c<p$. Let $\mathfrak{g}=\log (G)$ be the finite Lie ring associated to $G$ by the Lazard correspondence. We associate to $G$ a subset $\mathcal{S}(G)$ of $\mathfrak{g} / \mathfrak{z} \times \widehat{\mathfrak{g}^{\prime}}$, where $\mathfrak{z}$ denotes the centre of $\mathfrak{g}$ and $\widehat{\mathfrak{g}^{\prime}}=\operatorname{Hom}_{\mathbb{Z}}\left(\mathfrak{g}^{\prime}, \mathbb{C}^{\times}\right)$the Pontryagin dual of the derived Lie ring $\mathfrak{g}^{\prime}$. In Theorem $\mathrm{A}$ we show that the class and conjugacy vectors of $G$ may be described in terms of the cardinalities of fibres of the natural projections from $\mathcal{S}(G)$ onto $\mathfrak{g} / \mathfrak{z}$ and $\widehat{\mathfrak{g}^{\prime}}$.

Theorem $[\mathrm{B}$ gives a geometric description of the class and character vectors of certain $p$-groups and describes the variation of these vectors under 'extension of scalars'. More precisely, let $\mathfrak{o}$ be a compact discrete valuation ring of characteristic zero with residue field $\mathbf{k}$ of characteristic $p$. Theorem $\mathrm{B}$ asserts that if $\mathfrak{g}$ is a finite, nilpotent $\mathfrak{o}$-Lie algebra of class $c<p$, and $\mathfrak{g}^{\prime}$ or, equivalently, $\mathfrak{g} / \mathfrak{z}$, is a $\mathbf{k}$-vector space, then computing class and character vectors of the $p$-group $\exp (\mathfrak{g})$ associated to $\mathfrak{g}$ under the Lazard correspondence is equivalent to enumerating $\mathbf{k}$-rational points of degeneracy loci of certain 'commutator matrices' associated with $\mathfrak{g}$. Moreover, the formulae given in Theorem $B$ are uniformly valid for groups of the form $\exp \left(\mathfrak{g} \otimes_{\mathfrak{o}} \mathfrak{O}\right)$, where $\mathfrak{O}$ is a finite, unramified extension of $\mathfrak{o}$.

The Lie algebra $\mathfrak{g}$ may be obtained by base change from a globally defined object, such as a nilpotent $\mathbb{Z}$-Lie algebra. For some of the groups obtained from such Lie algebras, Theorem $B$ yields formulae which are uniform under variation of both the cardinality and the characteristic of the residue field. Consider, for instance, the free $\mathbb{F}_{q}$-Lie algebras $\mathfrak{f}_{r, c}\left(\mathbb{F}_{q}\right)$ on $r$ generators and of nilpotency class $c$, where $\mathbb{F}_{q}$ is a finite field of characteristic $p>c$. These algebras are of the form $\mathfrak{f}_{r, c}\left(\mathbb{F}_{q}\right)=\mathfrak{f}_{r, c}(\mathbb{Z}) \otimes_{\mathbb{Z}} \mathbb{F}_{q}$, where $\mathfrak{f}_{r, c}(\mathbb{Z})$ is the free nilpotent $\mathbb{Z}$-Lie algebra of class $c$ on $r$ generators. Theorem $\mathrm{B}$ applies to the groups $F_{r, c}\left(\mathbb{F}_{q}\right):=\exp \left(\mathfrak{f}_{r, c}\left(\mathbb{F}_{q}\right)\right)$.

In Section 2 we state Theorems $\mathrm{A}$ and $\mathrm{B}$ together with some applications to groups of the form $F_{r, c}\left(\mathbb{F}_{q}\right)$. Our main tools are the Lazard correspondence for $p$-groups of nilpotency class $c<p$ and the Kirillov orbit method for such groups. In Section 3 we review these tools and use them to prove Theorems A and B. In Section 4 we apply these results to uniformize a number of existing enumerative results on classes and characters of $p$-groups. In Section 5 we prove new results for the groups $F_{r, c}\left(\mathbb{F}_{q}\right)$, including those stated in Section 2. They extend and generalize results of Ito and Mann [19] for the relatively free groups of exponent $p$.

1.1. Notation. We denote the cardinality of a set $S$ by either $\# S$ or $|S|$. We write $\mathbb{N}$ for the set $\{1,2, \ldots\}$ of natural numbers. For $I \subseteq \mathbb{N}$ and $c \in \mathbb{R}$, we write $I_{0}$ for $I \cup\{0\}$ and $c I_{0}$ for $\left\{c i: i \in I_{0}\right\}$. Given $a, b \in \mathbb{N}_{0}$ we define $[a]=\{1, \ldots, a\}$ and $[a, b]=\{a, \ldots, b\}$. For $x \in \mathbb{R}$ we set $\lfloor x\rfloor:=\max \{m \in \mathbb{Z}: m \leq x\}$. If $I$ is any ordered set, then we write $I=\left\{i_{1}, \ldots, i_{l}\right\}_{<}$to indicate that $i_{1}<\cdots<i_{l}$. Given a proposition $P$, the 'Kronecker delta' $\delta_{P}$ is 1 if $P$ holds and 0 otherwise. If $n_{1}, \ldots, n_{r} \in \mathbb{N}_{0}$ and $f \in \mathbb{N}$, we write $\left(n_{1}, \ldots, n_{r}\right)_{f}$ for the vector

$$
(n_{1}, \underbrace{0, \ldots, 0}_{f-1}, n_{2}, \underbrace{0, \ldots, 0}_{f-1}, \ldots, n_{r}, \underbrace{0, \ldots, 0}_{f-1}) \in \mathbb{N}_{0}^{f r} ;
$$

if $f=1$ we drop the subscript. 
Given a ring $R$, an $R$-Lie algebra $\mathfrak{g}$ is an $R$-algebra with a 'Lie bracket', that is to say an $R$-bilinear map [,] $: \mathfrak{g} \times \mathfrak{g} \rightarrow \mathfrak{g}$ which is skew-symmetric and satisfies the Jacobi identity. A Lie ring is a $\mathbb{Z}$-Lie algebra. We write $[u, v, w, \ldots]$ for the left-normed Lie product $[\ldots[[u, v], w] \ldots] \in L$, and $\left[u,{ }_{i} v\right]$ denotes the Lie product $[u, v, \ldots, v]$ with $i$ occurrences of $v$.

Throughout this paper, $\mathfrak{o}$ is a compact discrete valuation ring of characteristic zero, viz. a finite extension of the $p$-adic integers $\mathbb{Z}_{p}$, with maximal ideal $\mathfrak{p}$ and residue field $\mathbf{k}=\mathfrak{o} / \mathfrak{p}$ of characteristic $p$. An arbitrary field is denoted by $\mathbb{K}$.

The centre and derived group of a group $G$ are denoted by $Z$ (or $Z(G)$ ) and $G^{\prime}$ respectively; the centre and derived ring of a Lie algebra $\mathfrak{g}$ are $\mathfrak{z}$ (or $Z(\mathfrak{g}))$ and $\mathfrak{g}^{\prime}$. We write [,] also for the induced map $\mathfrak{g} / \mathfrak{z} \times \mathfrak{g} / \mathfrak{z} \rightarrow \mathfrak{g}^{\prime},(x+\mathfrak{z}, y+\mathfrak{z}) \mapsto[x, y]$. Given $g \in G$ and $x \in \mathfrak{g}$ we write $C_{G}(g)$ and $C_{\mathfrak{g}}(x)$ for the respective centralizers.

Given a ring $R$ and integers $m$ and $n$, we write $\operatorname{Mat}(n \times m, R)$ for the $n \times m$ matrices over $R$. We abbreviate $\operatorname{Mat}(n \times n, R)$ to $\operatorname{Mat}(n, R)$. We denote the transpose of a matrix $A$ by $A^{\text {tr }}$.

By a character of a group we always mean a complex irreducible character.

\section{The MAIN RESUltS}

The Lazard correspondence establishes an order-preserving one-to-one correspondence between finite $p$-groups of nilpotency class $c<p$ on the one hand and finite nilpotent Lie rings of $p$-power order and class $c<p$ on the other; cf. [23, Example 10.24]. More precisely, one may define a group operation on such a Lie ring $\mathfrak{g}$ by the formula

$$
u \star v:=\sum_{i \leq c} F_{i}(u, v), \quad u, v \in \mathfrak{g},
$$

where $F_{i}(X, Y)$ is the homogeneous part of degree $i$ of the Hausdorff series $F(X, Y)$, an element in the completion of the free $\mathbb{Q}$-Lie algebra on variables $X$ and $Y$; cf. [23, $\$ 9.2$ ]. Then $\exp (\mathfrak{g}):=(\mathfrak{g}, \star)$ is a $p$-group of class $c$. The theorem underlying the Lazard correspondence asserts that the isomorphism type of every $p$-group $G$ of class $c<p$ arises in this manner from a Lie ring $\mathfrak{g}$, unique up to isomorphism. We denote the map underlying a fixed isomorphism $\exp (\mathfrak{g}) \cong G$ by $\exp : \mathfrak{g} \rightarrow G$, and write $\log$ for its inverse. We write $\widehat{\mathfrak{g}^{\prime}}$ for the Pontryagin dual $\operatorname{Hom}_{\mathbb{Z}}\left(\mathfrak{g}^{\prime}, \mathbb{C}^{\times}\right)$of the finite abelian $p$-group $\mathfrak{g}^{\prime}$.

Theorem A. Let $G$ be a finite p-group of nilpotency class $c<p$ and let $\mathfrak{g}=\log (G)$ be the corresponding Lie ring. Define

$$
\mathcal{S}(G):=\left\{(x, \omega) \in \mathfrak{g} / \mathfrak{z} \times \widehat{\mathfrak{g}^{\prime}}: \omega([x, z])=1 \text { for all } z \in \mathfrak{g} / \mathfrak{z}\right\},
$$

with projections $\pi_{1}: \mathcal{S}(G) \rightarrow \mathfrak{g} / \mathfrak{z}$ and $\pi_{2}: \mathcal{S}(G) \rightarrow \widehat{\mathfrak{g}^{\prime}}$. For $i \geq 0$,

$$
\begin{aligned}
& \operatorname{cc}_{i}(G)=\#\left\{x \in \mathfrak{g} / \mathfrak{z}:\left|\pi_{1}^{-1}(x)\right|=p^{-i}\left|\widehat{\mathfrak{g}^{\prime}}\right|\right\}|Z(G)| p^{-i} \\
& \operatorname{ch}_{i}(G)=\#\left\{\omega \in \widehat{\mathfrak{g}^{\prime}}:\left|\pi_{2}^{-1}(\omega)\right|=p^{-2 i}|\mathfrak{g} / \mathfrak{z}|\right\}\left|G / G^{\prime}\right| p^{-2 i} .
\end{aligned}
$$

In particular, the class number $k(G)=|\mathcal{S}(G)||Z(G)|\left|G^{\prime}\right|^{-1}$.

For a certain family of groups, Theorem B exploits this result to provide a uniform description of the class and character vectors in terms of the numbers of rational points of rank varieties of matrices of linear forms. We now formulate this more precisely. 
Assume that $\mathfrak{o}$ is a compact discrete valuation ring of characteristic zero and residue characteristic $p$, and that $\mathfrak{g}$ is a finite, nilpotent $\mathfrak{o}$-Lie algebra of class $c<p$. Set

$$
a:=\operatorname{rk}_{\mathfrak{o}}(\mathfrak{g} / \mathfrak{z}), \quad b:=\operatorname{rk}_{\mathfrak{o}}\left(\mathfrak{g}^{\prime}\right)
$$

and fix an ordered set $\mathbf{e}=\left(e_{1}, \ldots, e_{a}\right)$ of $\mathfrak{o}$-module generators for $\mathfrak{g} / \mathfrak{z}$ and an ordered set $\mathbf{f}=\left(f_{1}, \ldots, f_{b}\right)$ of $\mathfrak{o}$-module generators for $\mathfrak{g}^{\prime}$. We choose 'structure constants' $\lambda_{i j}^{k} \in \mathfrak{o}$ such that

$$
\left[e_{i}, e_{j}\right]=\sum_{k=1}^{b} \lambda_{i j}^{k} f_{k} \quad \text { and } \quad \lambda_{i j}^{k}=-\lambda_{j i}^{k}
$$

for all $i, j \in[a], k \in[b]$.

Definition 2.1. Let $\mathbf{X}=\left(X_{1}, \ldots, X_{a}\right)$ and $\mathbf{Y}=\left(Y_{1}, \ldots, Y_{b}\right)$ be independent variables. We define commutator matrices (with respect to $\mathbf{e}$ and $\mathbf{f}$ ) of $\mathfrak{o}$-linear forms in $\mathbf{X}$ and $\mathbf{Y}$, namely

$$
\begin{gathered}
A(\mathbf{X}) \in \operatorname{Mat}(a \times b, \mathfrak{o}[\mathbf{X}]), \text { where } A(\mathbf{X})_{i k}:=\sum_{j=1}^{a} \lambda_{i j}^{k} X_{j}, \quad i \in[a], k \in[b], \\
B(\mathbf{Y}) \in \operatorname{Mat}(a, \mathfrak{o}[\mathbf{Y}]), \text { where } B(\mathbf{Y})_{i j}:=\sum_{k=1}^{b} \lambda_{i j}^{k} Y_{k}, \quad i, j \in[a] .
\end{gathered}
$$

If $\mathfrak{g}$ is a $\mathbb{K}$-algebra with $\mathbb{K}$-basis $\mathcal{B}=\left(e_{1}, \ldots, e_{h}\right)$ such that the residue classes of the elements $e_{1}, \ldots, e_{a}$ form a $\mathbb{K}$-basis $\mathbf{e}$ for $\mathfrak{g} / \mathfrak{z}$ and $\mathbf{f}=\left(e_{h-b+1}, \ldots, e_{h}\right)$ is a $\mathbb{K}$-basis for $\mathfrak{g}^{\prime}$, then we refer to the associated commutator matrices $A$ and $B$ as 'with respect to $\mathcal{B}^{\prime}$.

Remark 2.2. The commutator matrix $B$ is clearly skew-symmetric. Hence $\operatorname{det}(B)$ is a square in $\mathfrak{o}[\mathbf{Y}]$, whose square root $\operatorname{Pf}(B):=\sqrt{\operatorname{det}(B)}$ is the Pfaffian of $B$. If $a$ is odd, then $\operatorname{Pf}(B)=0$.

Assume now that $\mathfrak{g} / \mathfrak{z}$ or, equivalently, $\mathfrak{g}^{\prime}$ is annihilated by $\mathfrak{p}$, the maximal ideal of $\mathfrak{o}$. We write $\mathbf{k}$ for the residue field $\mathfrak{o} / \mathfrak{p}$ of characteristic $p$. The set of generators $\mathbf{f}$ for $\mathfrak{g}^{\prime}$ may be regarded as a $\mathbf{k}$-basis for the $\mathbf{k}$-vector space $\mathfrak{g}^{\prime}$. Similarly, we view $\mathbf{e}$ as a $\mathbf{k}$-basis for the $\mathbf{k}$-vector space $\mathfrak{g} / \mathfrak{z}$.

The commutator matrices $A$ and $B$ may be considered as matrices of linear forms over $\mathbf{k}$. Let $\mathbb{K}$ be an extension of $\mathbf{k}$. For $\boldsymbol{x}=\left(x_{1}, \ldots, x_{a}\right) \in \mathbb{K}^{a}$ we write $A(\boldsymbol{x}) \in \operatorname{Mat}(a \times b, \mathbb{K})$ for the matrix obtained by evaluating the variables $X_{i}$ at $x_{i}$. Likewise $B(\mathbf{y}) \in \operatorname{Mat}(a, \mathbb{K})$ is defined for $\mathbf{y}=\left(y_{1}, \ldots, y_{b}\right) \in \mathbb{K}^{b}$. We note that the ranks of matrices of the form $B(\mathbf{y})$, for $\mathbf{y} \in \mathbb{K}^{b}$, are even integers.

It is well known that every finite field $\mathbf{k}$ is self-dual, i.e. (noncanonically) isomorphic to its Pontryagin dual. Indeed, let $\psi: \mathbf{k} \rightarrow \mathbb{C}^{\times}$be a nontrivial additive character of $\mathbf{k}$. For $a \in \mathbf{k}$ define $\psi_{a}(x)=\psi(a x)$ for $x \in \mathbf{k}$. The map $a \mapsto \psi_{a}$ is an isomorphism between $\mathbf{k}$ and its Pontryagin dual $\widehat{\mathbf{k}}$; cf., for instance, 4 . Since $\mathfrak{g}^{\prime} \cong \mathbf{k}^{b}$, this yields an isomorphism between $\mathfrak{g}^{\prime}$ and its dual $\widehat{\mathfrak{g}^{\prime}}$. On the other hand there is, of course, a - likewise noncanonical - isomorphism between $\mathfrak{g}^{\prime}$ and its linear dual $\operatorname{Hom}_{\mathbf{k}}\left(\mathfrak{g}^{\prime}, \mathbf{k}\right)$. We fix an isomorphism $\psi_{1}: \widehat{\mathfrak{g}}^{\prime} \rightarrow \operatorname{Hom}_{\mathbf{k}}\left(\mathfrak{g}^{\prime}, \mathbf{k}\right)$. The dual $\mathbf{k}$-basis $\mathbf{f}^{\vee}=\left(f_{k}^{\vee}\right)$ for $\operatorname{Hom}_{\mathbf{k}}\left(\mathfrak{g}^{\prime}, \mathbf{k}\right)$ gives a coordinate system

$$
\psi_{2}: \operatorname{Hom}_{\mathbf{k}}\left(\mathfrak{g}^{\prime}, \mathbf{k}\right) \rightarrow \mathbf{k}^{b}, \quad y=\sum_{k=1}^{b} y_{k} f_{k}^{\vee} \mapsto \mathbf{y}=\left(y_{1}, \ldots, y_{b}\right) .
$$


Set $\psi:=\psi_{2} \circ \psi_{1}: \widehat{\mathfrak{g}}^{\prime} \rightarrow \mathbf{k}^{b}$. Similarly, the $\mathbf{k}$-basis $\mathbf{e}$ for $\mathfrak{g} / \mathfrak{z}$ gives a coordinate system

$$
\varphi: \mathfrak{g} / \mathfrak{z} \rightarrow \mathbf{k}^{a}, \quad x=\sum_{j=1}^{a} x_{j} e_{j} \mapsto \boldsymbol{x}=\left(x_{1}, \ldots, x_{a}\right) .
$$

For a finite extension $\mathfrak{O}$ of $\mathfrak{o}$, we write $\mathfrak{g}(\mathfrak{O})$ for $\mathfrak{g} \otimes_{\mathfrak{o}} \mathfrak{O}$ and $\mathfrak{z}(\mathfrak{O})$ for $\mathfrak{z} \otimes_{\mathfrak{o}} \mathfrak{O}$. By tensoring, the bases associated with $\mathfrak{g}$ yield corresponding bases associated with $\mathfrak{g}(\mathfrak{O})$; we continue to write $\mathbf{e}$ for the $\mathfrak{O}$-basis $\mathbf{e} \otimes_{\mathfrak{o}} 1$ for $\mathfrak{g}(\mathfrak{O}) / \mathfrak{z}(\mathfrak{O})$, and likewise $\mathbf{f}$ for the $\mathfrak{O}$-basis $\mathbf{f} \otimes_{\mathfrak{o}} 1$ of $\mathfrak{g}(\mathfrak{O})^{\prime}$. Note that the commutator matrices $A$ and $B$ remain unchanged.

Assume further that $\mathfrak{O}$ is an unramified extension of $\mathfrak{o}$, with maximal ideal $\mathfrak{P}$. We identify the residue field $\mathfrak{O} / \mathfrak{P}$, a finite extension of $\mathbf{k}$, with $\mathbb{F}_{q}$. The $\mathfrak{O}$-Lie algebra $\mathfrak{g}(\mathfrak{O})$ inherits the property that the derived algebra and the cocentre of $\mathfrak{g}(\mathfrak{O})$ are annihilated by $\mathfrak{P}$. We consider e and $\mathbf{f}$ as $\mathbb{F}_{q}$-bases for the respective

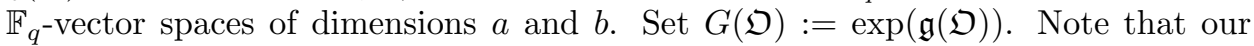
assumption on $\mathfrak{g}$ implies that both $G(\mathfrak{O})^{\prime}$ and $G(\mathfrak{O}) / Z(G(\mathfrak{O})$ ) have exponent $p$. Our second main result gives a uniform description of the set $\mathcal{S}(G(\mathfrak{O})$ ) introduced in Theorem $\mathrm{A}$ - and therefore for the class and character vectors of $G(\mathfrak{O})$ - in terms of the numbers of $\mathbb{F}_{q}$-rational points of degeneracy loci of the commutator matrices $A$ and $B$.

Theorem B. Let $\mathfrak{o}$ be a compact discrete valuation ring of characteristic zero and residue field $\mathbf{k}$ of characteristic $p$, and let $\mathfrak{g}$ be a finite, nilpotent $\mathfrak{o}$-Lie algebra of class $c<p$. Assume that $\mathfrak{g}^{\prime} \cong \mathbf{k}^{b}$ and that $\mathfrak{g} / \mathfrak{z} \cong \mathbf{k}^{a}$ as $\mathbf{k}$-vector spaces. Let $\mathfrak{O}$ be a finite, unramified extension of $\mathfrak{o}$, with residue field isomorphic to $\mathbb{F}_{q}$. The class sizes and character degrees of $G(\mathfrak{O})$ are powers of $q=p^{f}$. For $i \geq 0$,

$$
\begin{aligned}
& \operatorname{cc}_{i f}(G(\mathfrak{O}))=\#\left\{\boldsymbol{x} \in \mathbb{F}_{q}^{a}: \operatorname{rk}(A(\boldsymbol{x}))=i\right\}|Z(G(\mathfrak{O}))| q^{-i}, \\
& \operatorname{ch}_{i f}(G(\mathfrak{O}))=\#\left\{\mathbf{y} \in \mathbb{F}_{q}^{b}: \operatorname{rk}(B(\mathbf{y}))=2 i\right\}\left|G(\mathfrak{O}) / G(\mathfrak{O})^{\prime}\right| q^{-2 i} .
\end{aligned}
$$

We illustrate Theorem $\mathrm{B}$ with a well-known example.

Example 2.3. Let $G=U_{3}\left(\mathbb{F}_{q}\right)$ be the group of $3 \times 3$ upper-unitriangular matrices over $\mathbb{F}_{q}$, where $q=p^{f}$. Thus $|G|=q^{3}, a=2$ and $b=1$. For odd $p, G$ is isomorphic to $\exp \left(\mathfrak{f}_{2,2}\left(\mathbb{F}_{q}\right)\right)$, where $\mathfrak{f}_{2,2}\left(\mathbb{F}_{q}\right)$ is the $\mathbb{F}_{q}$-Lie algebra with $\mathbb{F}_{q}$-basis $(u, v, w)$, subject only to the relations $[v, u]=w,[u, w]=[v, w]=0$. With respect to this $\mathbb{F}_{q}$-basis

$$
A(\mathbf{X})=\left(\begin{array}{c}
-X_{2} \\
X_{1}
\end{array}\right) \quad \text { and } \quad B(\mathbf{Y})=\left(\begin{array}{cc} 
& -Y_{1} \\
Y_{1} &
\end{array}\right) .
$$

Theorem B confirms the well-known formulae $\operatorname{cc}(G)=\left(q, q^{2}-1\right)_{f}$ and $\operatorname{ch}(G)=$ $\left(q^{2}, q-1\right)_{f}$. We note that $\mathcal{S}(G)$ may be identified with $\left\{(u, v, w) \in \mathbb{F}_{q}^{3}: w u=w v=\right.$ $0\}$, showing that $k(G)=q^{2}+q-1$.

In Section 5 we study generalizations of the relatively free $p$-groups of exponent $p$. For integers $r \geq 2$ and $c \geq 1$ we consider the free $\mathbb{F}_{q}$-Lie algebra $\mathfrak{f}_{r, c}\left(\mathbb{F}_{q}\right)$ on $r$ generators and nilpotency class $c$, where $q=p^{f}$ is a power of a prime $p>c$. The Lazard correspondence associates the $p$-group $F_{r, c}\left(\mathbb{F}_{q}\right)=\exp \left(\mathfrak{f}_{r, c}\left(\mathbb{F}_{q}\right)\right)$ to this $\mathbb{F}_{q^{-}}$ Lie algebra. Our approach yields, for instance, a simple, geometric proof of the following generalization of [19, Theorem 5] and of Example 2.3. 
Proposition 2.4. Let $q=p^{f}$ be an odd prime power. The character degrees of $F_{r, 2}\left(\mathbb{F}_{q}\right)$ are $1, q, q^{2}, \ldots, q^{\lfloor r / 2\rfloor}$. For $0 \leq 2 i \leq r$

$$
\operatorname{ch}_{i f}\left(F_{r, 2}\left(\mathbb{F}_{q}\right)\right)=q^{r+i^{2}-3 i} \frac{\prod_{j=0}^{2 i-1}\left(q^{r-j}-1\right)}{\prod_{j=0}^{i-1}\left(q^{2(i-j)}-1\right)} .
$$

Proof. We fix an $\mathbb{F}_{q}$-basis $\left(x_{1}, \ldots, x_{r}, y_{k \ell}: 1 \leq k<\ell \leq r\right)$ for $\mathfrak{f}_{2, r}\left(\mathbb{F}_{q}\right)$, subject to the relations $\left[x_{\ell}, x_{k}\right]=y_{k \ell}$ for $1 \leq k<\ell \leq r$. Note that $a=r$ and $b=\left(\begin{array}{l}r \\ 2\end{array}\right)$. The commutator matrix $B(\mathbf{Y})$ with respect to this basis is the generic skew-symmetric matrix in variables $Y_{k \ell}$ for $1 \leq k<\ell \leq r$, so $B(\mathbf{Y})_{k \ell}=-Y_{k \ell}$. It is well known that, for $0 \leq 2 i \leq r$, the set $\left\{\mathbf{y} \in \mathbb{F}_{q}^{\left(\begin{array}{c}n \\ 2\end{array}\right)}: \operatorname{rk}(B(\mathbf{y}))=2 i\right\}$ has cardinality

$$
\nu_{i f}\left(F_{r, 2}\left(\mathbb{F}_{q}\right)\right):=q^{i(i-1)} \frac{\prod_{j=0}^{2 i-1}\left(q^{r-j}-1\right)}{\prod_{j=0}^{i-1}\left(q^{2(i-j)}-1\right)} ;
$$

see [6, Equation (7.5)]. TheoremBimplies that $\operatorname{ch}_{i f}\left(F_{r, 2}\left(\mathbb{F}_{q}\right)\right)=q^{r-2 i} \nu_{i f}\left(F_{r, 2}\left(\mathbb{F}_{q}\right)\right)$.

Recall that the 'Witt formula' is defined, for $i \in \mathbb{N}$, by

$$
W_{r}(i):=\frac{1}{i} \sum_{d \mid i} \mu(d) r^{i / d}
$$

where $\mu$ denotes the Möbius function; cf., for example, [12, Chapter 11]. We define

$$
n(r, c):= \begin{cases}\sum_{i=1}^{m} W_{r}(i) & \text { if } c=2 m+1, \\ \sum_{i=1}^{m-1} W_{r}(i)+\left\lfloor\frac{W_{r}(m)}{2}\right\rfloor & \text { if } c=2 m .\end{cases}
$$

Theorem 2.5. Assume that $(r, c) \neq(2,3)$, that $p>c$ and let $q$ be a power of $p$. The character degrees of $F_{r, c}\left(\mathbb{F}_{q}\right)$ are $1, q, q^{2}, \ldots, q^{n(r, c)}$.

The character vectors of the groups $F_{2,3}\left(\mathbb{F}_{q}\right)$ are given in Proposition [5.8. For $i \in[c]$ we define

$$
k(r, c, i):=-\delta_{i<(c+1) / 2}+\sum_{\ell=1}^{c-i} W_{r}(\ell) .
$$

Theorem 2.6. Assume $p>c$ and let $q$ be a power of $p$. The class sizes of $F_{r, c}\left(\mathbb{F}_{q}\right)$ are $q^{k(r, c, i)}$ for $1 \leq i \leq c$. For $j \geq 1$

$$
\operatorname{cc}_{j f}\left(F_{r, c}\left(\mathbb{F}_{q}\right)\right)=\sum_{\{i \in[c-1]: k(r, c, i)=j\}}\left(q^{W_{r}(i)}-1\right) q^{-j+\sum_{\ell=i+1}^{c} W_{r}(\ell)},
$$

and $\operatorname{cc}_{0}\left(F_{r, c}\left(\mathbb{F}_{q}\right)\right)=\left|Z\left(F_{r, c}\left(\mathbb{F}_{q}\right)\right)\right|=q^{W_{r}(c)}$.

Observe that the function $i \mapsto k(r, c, i)$ is injective unless $r=2$ and $c \in\{3,4\}$; in these cases the sum in (2.2) has at most two nonzero summands. Generically it has at most one.

Theorems 2.5 and 2.6 will be proven in Section 5

\section{Proofs of Theorems $\mathrm{A}$ and B}

The Lazard correspondence between $p$-groups and Lie rings of nilpotency class $c<p$ allows us to linearize the problem of enumerating conjugacy classes and characters. Let $G$ be a finite $p$-group of nilpotency class $c<p$, with associated Lie ring $\mathfrak{g}=\log (G)$. 
3.1. Counting conjugacy classes. It follows from straightforward calculations with the Hausdorff series that log induces an order-preserving correspondence between subgroups of $G$ and subalgebras of $\mathfrak{g}$, and log maps normal subgroups to ideals. In particular, $|G / Z|=|\mathfrak{g} / \mathfrak{z}|$ and $\left|G^{\prime}\right|=\left|\mathfrak{g}^{\prime}\right|$, and centralizers in $G$ correspond to centralizers in $\mathfrak{g}$. Thus

$$
\begin{aligned}
\operatorname{cc}_{i}(G) & =\#\left\{\text { conjugacy classes of } G \text { of cardinality } p^{i}\right\} \\
& =\#\left\{g \in G:\left|G: C_{G}(g)\right|=p^{i}\right\} p^{-i} \\
& =\#\left\{x \in \mathfrak{g}:\left|\mathfrak{g}: C_{\mathfrak{g}}(x)\right|=p^{i}\right\} p^{-i} \\
& =\#\left\{x \in \mathfrak{g} / \mathfrak{z}:\left|\mathfrak{g} / \mathfrak{z}: C_{\mathfrak{g} / \mathfrak{z}}(x)\right|=p^{i}\right\}|\mathfrak{z}| p^{-i} .
\end{aligned}
$$

The last equality reflects the fact that the centralizer of an element only depends on its coset modulo the centre. For $x \in \mathfrak{g} / \mathfrak{z}$ we define

$$
\begin{array}{rlrl}
\operatorname{ad}_{x}: \mathfrak{g} / \mathfrak{z} \rightarrow \mathfrak{g}^{\prime}, & z & \mapsto[z, x], \\
\operatorname{ad}_{x}^{\star}: \widehat{\mathfrak{g}^{\prime}} \rightarrow \widehat{\mathfrak{g} / \mathfrak{z}}, & \omega \mapsto \omega \circ \operatorname{ad}_{x} .
\end{array}
$$

Hence

$$
\begin{aligned}
\operatorname{cc}_{i}(G) & =\#\left\{x \in \mathfrak{g} / \mathfrak{z}:\left|\operatorname{im}\left(\operatorname{ad}_{x}\right)\right|=p^{i}\right\}|\mathfrak{z}| p^{-i} \\
& =\#\left\{x \in \mathfrak{g} / \mathfrak{z}:\left|\operatorname{ker}\left(\operatorname{ad}_{x}^{\star}\right)\right|=p^{-i}\left|\widehat{\mathfrak{g}^{\prime}}\right|\right\}|\mathfrak{z}| p^{-i}
\end{aligned}
$$

3.2. Kirillov's orbit method and counting characters. The Kirillov orbit method offers a linearization of the character theory of $G$ in terms of co-adjoint orbits: characters of $G$ correspond to orbits in $\widehat{\mathfrak{g}}:=\operatorname{Hom}_{\mathbb{Z}}\left(\mathfrak{g}, \mathbb{C}^{\times}\right)$, the Pontryagin dual of $\mathfrak{g}$, under the co-adjoint action $\mathrm{Ad}^{\star}$ of $G$ on $\widehat{\mathfrak{g}}$. The following is well known; see, for example, [5, Theorem 2.6] or [10, Theorem 4.4].

Theorem 3.1. Let $G=\exp (\mathfrak{g})$ be a finite p-group of nilpotency class $c<p$. Let $\Omega \subseteq \widehat{\mathfrak{g}}$ be a co-adjoint orbit and $\omega \in \Omega$.

(1) There exists a polarizing subalgebra $\mathfrak{h} \subseteq \mathfrak{g}$ for the bi-additive, skew-symmetric form $B_{\omega}: \mathfrak{g} \times \mathfrak{g} \rightarrow \mathbb{C}^{\times},(u, v) \mapsto \omega([u, v])$ : namely, a subalgebra $\mathfrak{h}$ that is maximal with respect to the property that $\left.B_{\omega}\right|_{\mathfrak{h} \times \mathfrak{h}} \equiv 1$. Setting

$$
\operatorname{Rad}\left(B_{\omega}\right):=\left\{u \in \mathfrak{g}: B_{\omega}(u, v)=1 \text { for all } v \in \mathfrak{g}\right\},
$$

$\exp \left(\operatorname{Rad}\left(B_{\omega}\right)\right)$ is the $\operatorname{Ad}^{\star}-$ stabilizer $\operatorname{Stab}_{G}(\omega)$, and $|\mathfrak{g}: \mathfrak{h}|=\left|\mathfrak{h}: \operatorname{Rad}\left(B_{\omega}\right)\right|$. Thus, with $H:=\exp (\mathfrak{h})$,

$$
|\Omega|^{1 / 2}=\left|G: \operatorname{Stab}_{G}(\omega)\right|^{1 / 2}=\left|\mathfrak{g}: \operatorname{Rad}\left(B_{\omega}\right)\right|^{1 / 2}=|\mathfrak{g}: \mathfrak{h}|=|G: H| .
$$

(2) Viewing $\omega$ as a function on $G$ (via $\log )$, the function $\left.\omega\right|_{H}$ is a one-dimensional representation of $H$. The induced representation $U_{\Omega}:=\operatorname{Ind}_{H}^{G} \omega$ of $G$ is irreducible, independent of $\omega$, and has dimension $|\Omega|^{1 / 2}$. All irreducible complex representations of $G$ have this form.

(3) The character of $U_{\Omega}$ is given by $|\Omega|^{-1 / 2} \sum_{\omega \in \Omega} \omega(g)$, for $g \in G$.

Remark 3.2. A Kirillov orbit method for torsion-free finitely generated nilpotent pro- $p$ groups of class 2 that holds for all primes $p$ is presented in [27, Section 2.4]. We expect that it can be used to prove the conclusions of Theorem 3.1 for 2-groups of class 2 . 
Theorem 3.1 reduces the problem of enumerating the characters of $G$ to that of computing the indices in $\mathfrak{g}$ of the radicals $\operatorname{Rad}\left(B_{\omega}\right)$, as $\omega$ ranges over $\widehat{\mathfrak{g}}$. In fact, given $\omega \in \widehat{\mathfrak{g}}$, the form $B_{\omega}$ only depends on the restriction of $\omega$ to $\mathfrak{g}^{\prime}$. Given $\omega \in \widehat{\mathfrak{g}^{\prime}}$ we therefore write $B_{\omega}$ for $B_{\widetilde{\omega}}$, where $\widetilde{\omega} \in \widehat{\mathfrak{g}}$ is any extension of $\omega$. With this notation, Theorem 3.1 implies that

$$
\begin{aligned}
\operatorname{ch}_{i}(G) & =\#\left\{\text { irreducible complex characters of } G \text { of degree } p^{i}\right\} \\
& =\#\left\{\text { co-adjoint orbits } \Omega \subseteq \widehat{\mathfrak{g}} \text { of size } p^{2 i}\right\} \\
& =\#\left\{\omega \in \widehat{\mathfrak{g}}:\left|\mathfrak{g}: \operatorname{Rad}\left(B_{\omega}\right)\right|=p^{2 i}\right\} p^{-2 i} \\
& =\#\left\{\omega \in \widehat{\mathfrak{g}^{\prime}}:\left|\mathfrak{g}: \operatorname{Rad}\left(B_{\omega}\right)\right|=p^{2 i}\right\}\left|\mathfrak{g} / \mathfrak{g}^{\prime}\right| p^{-2 i} \\
& =\#\left\{\omega \in \widehat{\mathfrak{g}^{\prime}}:\left|\operatorname{Rad}\left(B_{\omega}\right) / \mathfrak{z}\right|=p^{-2 i}|\mathfrak{g} / \mathfrak{z}|\right\}\left|\mathfrak{g} / \mathfrak{g}^{\prime}\right| p^{-2 i}
\end{aligned}
$$

3.3. Proof of Theorem $\mathbf{A}$, For $i \in \mathbb{N}_{0}$ we define

$$
\begin{aligned}
\mu_{i}(G) & :=\#\left\{x \in \mathfrak{g} / \mathfrak{z}:\left|\operatorname{ker}\left(\operatorname{ad}_{x}^{\star}\right)\right|=p^{-i}\left|\widehat{\mathfrak{g}^{\prime}}\right|\right\}, \\
\nu_{i}(G) & :=\#\left\{\omega \in \widehat{\mathfrak{g}^{\prime}}:\left|\operatorname{Rad}\left(B_{\omega}\right) / \mathfrak{z}\right|=p^{-2 i}|\mathfrak{g} / \mathfrak{z}|\right\} .
\end{aligned}
$$

Equations (3.1) and (3.2) imply that

$$
\operatorname{cc}_{i}(G)=\mu_{i}(G)|\mathfrak{z}| p^{-i} \quad \text { and } \quad \operatorname{ch}_{i}(G)=\nu_{i}(G)\left|\mathfrak{g} / \mathfrak{g}^{\prime}\right| p^{-2 i} .
$$

For $x \in \mathfrak{g} / \mathfrak{z}$ and $\omega \in \widehat{\mathfrak{g}^{\prime}}$, observe that $x \in \operatorname{Rad}\left(B_{\omega}\right) / \mathfrak{z}$ if and only if $\omega \in \operatorname{ker}\left(\operatorname{ad}_{x}^{\star}\right)$. Thus

$$
\begin{aligned}
\mathcal{S}(G) & =\left\{(x, \omega) \in \mathfrak{g} / \mathfrak{z} \times \widehat{\mathfrak{g}^{\prime}}: \omega([x, z])=1 \text { for all } z \in \mathfrak{g} / \mathfrak{z}\right\} \\
& =\left\{(x, \omega) \in \mathfrak{g} / \mathfrak{z} \times \widehat{\mathfrak{g}^{\prime}}: \omega \in \operatorname{ker}\left(\operatorname{ad}_{x}^{\star}\right)\right\} \\
& =\left\{(x, \omega) \in \mathfrak{g} / \mathfrak{z} \times \widehat{\mathfrak{g}}^{\prime}: x \in \operatorname{Rad}\left(B_{\omega}\right) / \mathfrak{z}\right\} .
\end{aligned}
$$

Using the natural projections $\pi_{1}: \mathcal{S}(G) \rightarrow \mathfrak{g} / \mathfrak{z}$ and $\pi_{2}: \mathcal{S}(G) \rightarrow \widehat{\mathfrak{g}^{\prime}}$, we see that

$$
\begin{aligned}
\mu_{i}(G) & =\#\left\{x \in \mathfrak{g} / \mathfrak{z}:\left|\pi_{1}^{-1}(x)\right|=p^{-i}\left|\widehat{\mathfrak{g}^{\prime}}\right|\right\}, \\
\nu_{i}(G) & =\#\left\{\omega \in \widehat{\mathfrak{g}^{\prime}}:\left|\pi_{2}^{-1}(\omega)\right|=p^{-2 i}|\mathfrak{g} / \mathfrak{z}|\right\} .
\end{aligned}
$$

We obtain two descriptions of the class number $k(G)$ :

$$
\begin{gathered}
\left|\mathfrak{g}^{\prime}\right| k(G)=\left|\mathfrak{g}^{\prime}\right| \sum_{i} \operatorname{cc}_{i}(G)=|\mathfrak{z}| \sum_{i} p^{-i}\left|\mathfrak{g}^{\prime}\right| \mu_{i}(G)=|\mathfrak{z}||\mathcal{S}(G)| \\
|\mathfrak{g} / \mathfrak{z}| k(G)=|\mathfrak{g} / \mathfrak{z}| \sum_{i} \operatorname{ch}_{i}(G)=\left|\mathfrak{g} / \mathfrak{g}^{\prime}\right| \sum_{i} p^{-2 i}|\mathfrak{g} / \mathfrak{z}| \nu_{i}(G)=\left|\mathfrak{g} / \mathfrak{g}^{\prime}\right||\mathcal{S}(G)| .
\end{gathered}
$$

We deduce that $k(G)=|\mathcal{S}(G)||\mathfrak{z}|\left|\mathfrak{g}^{\prime}\right|^{-1}=|\mathcal{S}(G)||Z(G)|\left|G^{\prime}\right|^{-1}$. This proves Theorem A

3.4. Proof of Theorem B, Recall that $\mathfrak{o}$ is a compact discrete valuation ring with residue field $\mathbf{k}=\mathfrak{o} / \mathfrak{p}$ of characteristic $p$, and that $\mathfrak{g}$ is a finite, nilpotent $\mathfrak{o}$-Lie algebra of class $c<p$ with the property that $\mathfrak{g} / \mathfrak{z}$ and $\mathfrak{g}^{\prime}$ are annihilated by $\mathfrak{p}$. Further recall the isomorphisms $\varphi: \mathfrak{g} / \mathfrak{z} \rightarrow \mathbf{k}^{a}$ and $\psi: \widehat{\mathfrak{g}}^{\prime} \rightarrow \mathbf{k}^{b}$ introduced in Section 2, Consider the $p$-group $G=\exp (\mathfrak{g})$. By (3.3),

$$
\mathcal{S}(G)=\left\{(x, \omega) \in \mathfrak{g} / \mathfrak{z} \times \widehat{\mathfrak{g}^{\prime}}: x \in \operatorname{Rad}\left(B_{\omega}\right) / \mathfrak{z}\right\} .
$$


The following lemma, proved analogously to [1, Lemma 3.3], characterizes membership of $\mathcal{S}(G)$ in terms of the above coordinate systems for $\mathfrak{g} / \mathfrak{z}$ and $\widehat{\mathfrak{g}^{\prime}}$.

Lemma 3.3. Let $x \in \mathfrak{g} / \mathfrak{z}$ and $\omega \in \widehat{\mathfrak{g}^{\prime}}$ correspond to $\varphi(x)=\boldsymbol{x} \in \mathbf{k}^{a}$ and $\psi(\omega)=$ $\mathbf{y} \in \mathbf{k}^{b}$. Then

$$
x \in \operatorname{Rad}\left(B_{\omega}\right) / \mathfrak{z} \text { if and only if } A(\boldsymbol{x}) \mathbf{y}^{\mathrm{tr}}=B(\mathbf{y}) \boldsymbol{x}^{\mathrm{tr}}=0 .
$$

Now let $\mathfrak{O}$ be a finite, unramified extension of $\mathfrak{o}$, with residue field isomorphic to $\mathbb{F}_{q}=\mathbb{F}_{p^{f}}$, say. Applying Theorem $\mathrm{A}$, (3.3) and Lemma 3.3 to $\mathfrak{g}(\mathfrak{O})=\mathfrak{g} \otimes_{\mathfrak{o}} \mathfrak{O}$ reduces the computation of the class and character vector of $G(\mathfrak{O})=\exp (\mathfrak{g}(\mathfrak{O}))$ to the problem of counting the solutions to linear equations over $\mathbb{F}_{q}$. In particular, all class sizes and character degrees are powers of $q=p^{f}$. For $i \geq 0$,

$$
\begin{aligned}
& \mu_{i f}(G(\mathfrak{O}))=\#\left\{\boldsymbol{x} \in \mathbb{F}_{q}^{a}: \operatorname{rk}(A(\boldsymbol{x}))=i\right\}, \\
& \nu_{i f}(G(\mathfrak{O}))=\#\left\{\mathbf{y} \in \mathbb{F}_{q}^{b}: \operatorname{rk}(B(\mathbf{y}))=2 i\right\} .
\end{aligned}
$$

This proves Theorem $\mathrm{B}$.

\section{Consequences of Theorems A And B}

4.1. Isoclinism. Recall from 11 that two p-groups $G_{1}$ and $G_{2}$ are isoclinic if there are isomorphisms $\theta: G_{1} / Z_{1} \rightarrow G_{2} / Z_{2}$ and $\varphi: G_{1}^{\prime} \rightarrow G_{2}^{\prime}$ such that, for all $\alpha, \beta \in G_{1}^{\prime}, \varphi([\alpha, \beta])=\left[\theta\left(\alpha Z_{1}\right), \theta\left(\beta Z_{1}\right)\right]$. The pair $(\theta, \varphi)$ is an isoclinism between $G_{1}$ and $G_{2}$.

If $G_{1}$ and $G_{2}$ have nilpotency class less than $p$ and $(\theta, \varphi)$ is an isoclinism between $G_{1}=\exp \left(\mathfrak{g}_{1}\right)$ and $G_{2}=\exp \left(\mathfrak{g}_{2}\right)$, then there is a pair of associated maps $\left(\Theta, \Phi^{-1}\right)$, where $\Theta=\log \circ \theta \circ \exp , \Phi^{-1}=\log \circ \varphi^{-1} \circ \exp$ and $\widehat{\Phi^{-1}}: \widehat{\mathfrak{g}_{1}} \rightarrow \widehat{\mathfrak{g}_{2}}, \omega_{1} \mapsto \omega_{1} \circ \Phi^{-1}$. The isoclinism $(\theta, \varphi)$ induces a bijection $\left(\Theta, \widehat{\Phi^{-1}}\right): \mathcal{S}\left(G_{1}\right) \rightarrow \mathcal{S}\left(G_{2}\right)$, where, for $i \in\{1,2\}, \mathcal{S}\left(G_{i}\right)$ are as defined in Theorem $\mathrm{A}$ in particular,

$$
\mathcal{S}\left(G_{1}\right)=\left\{\left(x_{1}, \omega_{1}\right) \in\left(\mathfrak{g}_{1} / Z\left(\mathfrak{g}_{1}\right)\right) \times \widehat{\mathfrak{g}_{1}^{\prime}}: \omega_{1}\left(\left[x_{1}, z_{1}\right]\right)=1 \text { for all } z_{1} \in \mathfrak{g}_{1} / Z\left(\mathfrak{g}_{1}\right)\right\} .
$$

By definition, $\omega_{1}\left(\left[x_{1}, z_{1}\right]\right)=1$ if and only if $\omega_{1}\left(\Phi^{-1}\left(\left[\Theta\left(x_{1}\right), \Theta\left(z_{1}\right)\right]\right)\right)=1$; this holds if and only if $\widehat{\Phi^{-1}}\left(\omega_{1}\right)\left(\left[\Theta\left(x_{1}\right), \Theta\left(z_{1}\right)\right]\right)=1$. Therefore $\left(\Theta, \widehat{\Phi^{-1}}\right)\left(\mathcal{S}\left(G_{1}\right)\right)=$ $\mathcal{S}\left(G_{2}\right)$. This, of course, merely reflects the well-known fact that isoclinic groups have, up to multiplication by $p$-powers, identical class (and character) vectors.

4.2. Pfaffian hypersurfaces. Boston and Isaacs 3 studied the class vectors of some $p$-groups of class 2 and exponent $p$. In this section we prove a generalization and extension of [3, Theorem 3.2]. We first describe our broader context. Let $\mathfrak{o}$ be a compact, discrete valuation ring with residue field $\mathfrak{o} / \mathfrak{p}$, which we identify with $\mathbb{F}_{q}$, where $q=p^{f}$ is an odd prime power. Let $\mathfrak{g}$ be a finite, nilpotent $\mathfrak{o}$-Lie algebra of class 2 . Assume that $\mathfrak{g} / \mathfrak{z}$ and $\mathfrak{g}^{\prime}$ are annihilated by $\mathfrak{p}$, so that Theorem B applies. The coordinate systems introduced in Section 2 identify $\mathfrak{g} / \mathfrak{z}$ with $\mathbb{F}_{q}^{a}$ and $\mathfrak{g}^{\prime}$ with $\mathbb{F}_{q}^{b}$, where we write $a$ for the $\mathfrak{o}$-rank of $\mathfrak{g} / \mathfrak{z}$ and $b$ for the $\mathfrak{o}$-rank of $\mathfrak{g}^{\prime}$. Recall from Definition 2.1 the commutator matrix $B$ associated to $\mathfrak{g}$ with respect to the chosen bases. We denote by $\mathbb{P}^{b-1}\left(\mathbb{F}_{q}\right)$ the $(b-1)$-dimensional projective space over $\mathbb{F}_{q}$. Note that $\operatorname{rk}(B(\widetilde{\mathbf{y}}))$ is well defined for $\widetilde{\mathbf{y}}=\left(\widetilde{y_{1}}: \cdots: \widetilde{y_{b}}\right) \in \mathbb{P}^{b-1}\left(\mathbb{F}_{q}\right)$. We write $G=\exp (\mathfrak{g})$ and recall that, by Theorem $\mathrm{B}, \operatorname{cs}(G)$ and $\operatorname{ch}(G)$ consist of powers of $q=p^{f}$. 
Theorem 4.1. Assume that $a>2$,

$$
\left\{\operatorname{rk}(B(\widetilde{\mathbf{y}})): \widetilde{\mathbf{y}} \in \mathbb{P}^{b-1}\left(\mathbb{F}_{q}\right)\right\}=\{a-2, a\}
$$

and that, for every line $L \subset \mathbb{P}^{b-1}\left(\mathbb{F}_{q}\right)$, there exists $\widetilde{\mathbf{y}} \in L$ such that $\operatorname{rk}(B(\widetilde{\mathbf{y}}))=a$. Let

$$
n:=\#\left\{\widetilde{\mathbf{y}} \in \mathbb{P}^{b-1}\left(\mathbb{F}_{q}\right): \operatorname{rk}(B(\widetilde{\mathbf{y}}))=a-2\right\} .
$$

Then

$$
\begin{gathered}
\operatorname{cc}_{i f}(G)= \begin{cases}|Z| & \text { if } i=0, \\
|Z| q^{-b+1} n\left(q^{2}-1\right) & \text { if } i=b-1, \\
|Z| q^{-b}\left(q^{a}-1-n\left(q^{2}-1\right)\right) & \text { if } i=b, \\
0 & \text { otherwise, }\end{cases} \\
\operatorname{ch}_{i f}(G)= \begin{cases}\left|G / G^{\prime}\right| & \text { if } i=0, \\
\left|G / G^{\prime}\right| q^{-a+2} n(q-1) & \text { if } i=a / 2-1, \\
\left|G / G^{\prime}\right| q^{-a}\left(q^{b}-1-n(q-1)\right) & \text { if } i=a / 2, \\
0 & \text { otherwise. }\end{cases}
\end{gathered}
$$

In particular

$$
k(G)=|G|\left(q^{-a}+q^{-b}+q^{-a-b}\left(n\left(q^{2}-1\right)(q-1)-1\right)\right) .
$$

Remark 4.2. Geometrically, the hypotheses of Theorem 4.1 imply that the projective Pfaffian hypersurface defined by $\operatorname{Pf}(B)$ contains no lines over $\mathbb{F}_{q}$. In particular, the Pfaffian is not identically zero, and thus $a$ is even; cf. Remark 2.2. Hypothesis (4.1) implies that $b>1$ and is satisfied if (but not only if) the Pfaffian defines a smooth hypersurface in $\mathbb{P}^{b-1}\left(\mathbb{F}_{q}\right)$; cf. [31, Lemma 5].

Proof. Remark 4.2 shows that $a$ is even. To prove (4.3) we observe that, by the hypotheses,

$$
\nu_{i f}(G)= \begin{cases}1 & \text { if } i=0 \\ n(q-1) & \text { if } i=a / 2-1 \\ q^{b}-1-n(q-1) & \text { if } i=a / 2 \\ 0 & \text { otherwise }\end{cases}
$$

The claim about $\operatorname{ch}(G)$ then follows from Theorem $\mathrm{B}$ which asserts that $\operatorname{ch}_{i f}(G)=$ $\nu_{i f}(G)\left|G / G^{\prime}\right| q^{-2 i}$ for $i \in[a / 2]_{0}$.

To prove (4.2) it suffices to show that, first, $\mu_{i f}(G)=0$ for $i \in[b-2]$ and, second, $\mu_{(b-1) f}(G)=n\left(q^{2}-1\right)$. Indeed, clearly $\mu_{0}(G)=1$ and $\sum_{i=0}^{b} \mu_{i f}(G)=q^{a}$, so that $\mu_{b f}(G)=q^{a}-1-n\left(q^{2}-1\right)$. The claim about $\operatorname{cc}(G)$ then follows from Theorem $\mathrm{B}$ which asserts that $\operatorname{cc}_{i f}(G)=\mu_{i f}(G)|Z| q^{-i}$ for $i \in[b]_{0}$.

Given $\mathbf{y} \in \mathbb{F}_{q}^{b}$ we view $B(\mathbf{y})$ as the matrix of an endomorphism of $\mathbb{F}_{q}^{a}$, whose kernel we denote by $\operatorname{ker}(B(\mathbf{y}))$. Likewise, given $\boldsymbol{x} \in \mathbb{F}_{q}^{a}$, we view $A(\boldsymbol{x})$ as the matrix of the linear map $\mathbb{F}_{q}^{b} \rightarrow \mathbb{F}_{q}^{a}, \mathbf{y} \mapsto \mathbf{y} A(\boldsymbol{x})^{\mathrm{tr}}$, whose kernel we denote by $\operatorname{ker}(A(\boldsymbol{x}))$. 
Let $\mathbf{y} \in \mathbb{F}_{q}^{b}$ be one of the $n(q-1)$ elements with $\operatorname{rk}(B(\mathbf{y}))=a-2$, so $\operatorname{dim}(\operatorname{ker}(B(\mathbf{y})))$ $=2$. Observe that $\operatorname{rk}(A(\boldsymbol{x}))<b$ for all $\boldsymbol{x} \in \operatorname{ker}(B(\mathbf{y}))$. We claim that $\operatorname{rk}(A(\boldsymbol{x}))=$ $b-1$ for all such $\boldsymbol{x}$ which are nonzero. Indeed, assume that $\boldsymbol{x} \neq \mathbf{0}$ with $\operatorname{rk}(A(\boldsymbol{x})) \leq$ $b-2$. Let $V \leq \mathbb{F}_{q}^{b}$ be a 2-dimensional subspace of $\operatorname{ker}(A(\boldsymbol{x}))$. For every $\mathbf{y} \in V$ we deduce using Lemma 3.3 that $\mathbf{y} A(\boldsymbol{x})^{\operatorname{tr}}=\boldsymbol{x} B(\mathbf{y})^{\operatorname{tr}}=0$. Therefore $V$ defines a line in $\mathbb{P}^{b-1}\left(\mathbb{F}_{q}\right)$ on which no point $\widetilde{\mathbf{y}}$ satisfies $\operatorname{rk}(B(\widetilde{\mathbf{y}}))=a$, contradicting our hypotheses. Thus $\operatorname{rk}(A(\boldsymbol{x}))=b-1$. This shows that $\mu_{i f}(G)=0$ for $i \in[b-2]$, establishing the first claim.

Every $\mathbf{y} \in \mathbb{F}_{q}^{b} \backslash\{\mathbf{0}\}$ such that $\operatorname{rk}(B(\mathbf{y}))=a-2$ gives rise to $q^{2}-1$ elements $\boldsymbol{x} \in \mathbb{F}_{q}^{a} \backslash\{\mathbf{0}\}$ such that $\operatorname{rk}(A(\boldsymbol{x}))=b-1$, namely the nonzero elements of the 2-dimensional space $\operatorname{ker}(B(\mathbf{y}))$. Likewise, every $\boldsymbol{x} \in \mathbb{F}_{q}^{a} \backslash\{\mathbf{0}\}$ such that $\operatorname{rk}(A(\boldsymbol{x}))=$ $b-1$ gives rise to $q-1$ elements $\mathbf{y}$ with this property, namely the nonzero elements of its nullspace. Thus $\mu_{(b-1) f}(G)=n(q-1) \frac{\left(q^{2}-1\right)}{(q-1)}=n\left(q^{2}-1\right)$, establishing the second claim.

Example 4.3. Let $p$ be a prime and $\alpha \in \mathbb{F}_{p}^{\times}$. Let $\mathfrak{g}_{\alpha}$ be the 9 -dimensional nilpotent $\mathbb{F}_{p}$-Lie algebra of class 2 with $\mathbb{F}_{p}$-basis $\left(e_{1}, \ldots, e_{6}, f_{1}, f_{2}, f_{3}\right)$ subject only to the relations $\left[e_{1}, e_{4}\right]=f_{1},\left[e_{1}, e_{5}\right]=f_{2},\left[e_{1}, e_{6}\right]=\alpha f_{3},\left[e_{2}, e_{4}\right]=f_{3},\left[e_{2}, e_{5}\right]=f_{1}$, $\left[e_{2}, e_{6}\right]=f_{2},\left[e_{3}, e_{4}\right]=f_{3},\left[e_{3}, e_{6}\right]=f_{1}$, where $0 \neq \alpha \in \mathbb{F}_{p}$. With respect to this basis, the commutator matrices are:

$$
\begin{gathered}
A(\mathbf{X})=\left(\begin{array}{ccc}
-X_{4} & -\alpha X_{6} & -X_{5} \\
-X_{5} & -X_{4} & -X_{6} \\
-X_{6} & -X_{4} & 0 \\
X_{1} & X_{2}+X_{3} & 0 \\
X_{2} & 0 & X_{1} \\
X_{3} & \alpha X_{1} & X_{2}
\end{array}\right) ; \\
B(\mathbf{Y})=\left(\begin{array}{cc}
0 & U(\mathbf{Y}) \\
-U(\mathbf{Y})^{\operatorname{tr}} & 0
\end{array}\right), \text { where } U(\mathbf{Y})=\left(\begin{array}{ccc}
Y_{1} & Y_{2} & \alpha Y_{3} \\
Y_{3} & Y_{1} & Y_{2} \\
Y_{3} & 0 & Y_{1}
\end{array}\right) .
\end{gathered}
$$

Boston and Isaacs [3] study the groups $G_{\alpha}=\exp \left(\mathfrak{g}_{\alpha}\right)$, which satisfy the hypotheses of Theorem 4.1 if $p$ is odd. They prove that $k\left(G_{\alpha}\right)=p^{6}+p^{3}-1+n_{\alpha}\left(p^{2}-1\right)(p-1)$, where $n_{\alpha}=\#\left\{\widetilde{\mathbf{y}} \in \mathbb{P}^{2}\left(\mathbb{F}_{p}\right): \operatorname{rk}(B(\widetilde{\mathbf{y}}))=4\right\}$, which accords with Theorem 4.1. They also show that $\#\left\{n_{\alpha}: \alpha \in \mathbb{F}_{p}^{\times}\right\} \rightarrow \infty$ as $p \rightarrow \infty$. Thus they establish that the number of different values assumed by $k(G)$ as $G$ runs over all groups of order $p^{9}$ tends to infinity with $p$.

Example 4.4. Let $\mathfrak{g}$ be the 8-dimensional nilpotent $\mathbb{F}_{q}$-Lie algebra of class 2 with $\mathbb{F}_{q}$-basis $\left(e_{1}, \ldots, e_{4}, f_{1}, \ldots, f_{4}\right)$ subject only to the relations $\left[e_{1}, e_{3}\right]=f_{1},\left[e_{1}, e_{4}\right]=$ $f_{2},\left[e_{2}, e_{3}\right]=f_{3},\left[e_{2}, e_{4}\right]=f_{4}$. The class and character vectors of $G=\exp (\mathfrak{g})$ are the following:

$$
\begin{aligned}
\left(\operatorname{cc}_{i f}(G)\right)_{i \in\{0,1,2,3\}} & =\left(q^{4}, 0,2\left(q^{2}-1\right) q^{2}, q\left(q^{2}-1\right)^{2}\right)_{f} \\
\left(\operatorname{ch}_{i f}(G)\right)_{i \in\{0,1,2\}} & =\left(q^{4}, q^{2}(q-1)(q+1)^{2}, q^{4}-1-(q+1)^{2}(q-1)\right)_{f} .
\end{aligned}
$$


This follows from inspection of the commutator matrices

$$
A(\mathbf{X})=\left(\begin{array}{cccc}
X_{3} & X_{4} & & \\
& & X_{3} & X_{4} \\
-X_{1} & & -X_{2} & \\
& -X_{1} & & -X_{2}
\end{array}\right), \quad B(\mathbf{Y})=\left(\begin{array}{rrrr} 
& & Y_{1} & Y_{2} \\
& Y_{3} & Y_{4} \\
-Y_{1} & -Y_{3} & & \\
-Y_{2} & -Y_{4} & &
\end{array}\right) .
$$

The class vector differs from (4.2), but the character vector agrees with (4.3). The hypothesis of Theorem4.1 regarding lines in $\mathbb{P}^{3}\left(\mathbb{F}_{q}\right)$ is not satisfied. We observe that the factor $2(q+1)$ of $\operatorname{cc}_{2 f}(G)$ is the number of lines on the Pfaffian hypersurface, the quadric surface defined by $Y_{1} Y_{4}-Y_{2} Y_{3}=0$.

4.3. Prescribing class sizes and character degrees. It is known that every finite set of $p$-powers containing 1 can be realized as the class sizes or character degrees of a finite $p$-group; cf. [7] and [17] respectively. Such results can be obtained readily using Theorems $\mathrm{A}$ and $\mathrm{B}$. Throughout this section let $p$ be an odd prime.

4.3.1. We show how to obtain the result of [17. Let $I \subset \mathbb{N}$ be finite and let $j=$ $\max (I)$. To construct a $p$-group $G$ such that $\operatorname{cd}(G)=\left\{p^{i}: i \in I_{0}\right\}$, consider the $\mathbb{F}_{p}$-Lie algebra $\mathfrak{g}$, with $\mathbb{F}_{p}$-basis consisting of $x_{1}, \ldots, x_{2 j}$ and $y_{i}$ for $i \in I$, subject only to the relations

$$
\left[x_{r}, x_{t}\right]=\left\{\begin{array}{ll}
y_{i} & \text { if } t-r=i \in I, \\
0 & \text { otherwise, }
\end{array} \quad \text { for } r, t \in[2 j] .\right.
$$

The commutator matrix $B(\mathbf{Y}) \in \operatorname{Mat}\left(2 j, \mathbb{F}_{p}[\mathbf{Y}]\right)$ in variables $Y_{i}$ for $i \in I$ with respect to this basis is the sum of the $(2 j \times 2 j)$-matrices

$$
\left(\begin{array}{ccc}
0 & Y_{i} \operatorname{Id}_{i} & \\
-Y_{i} \operatorname{Id}_{i} & 0 & \\
& & 0_{2(j-i)}
\end{array}\right) \text {, where } i \in I .
$$

Clearly $\left\{\operatorname{rk}(B(\mathbf{y})): \mathbf{y} \in \mathbb{F}_{p}^{|I|}\right\}=I_{0}$. Theorem Bimplies that $\operatorname{cd}(\exp (\mathfrak{g}))=\left\{p^{i}: i \in\right.$ $\left.I_{0}\right\}$.

4.3.2. Fernández-Alcober and Moretó 9 prove that for every two integers $u, v>1$ there exists a finite $p$-group $H$ of class 2 such that $|\operatorname{cd}(H)|=u$ and $|\operatorname{cs}(H)|=v$. As part of their proof, they construct, for given $l, n \in \mathbb{N}$, a $p$-group $G$ with $\operatorname{cd}(G)=$ $\left\{1, p^{l}\right\}$, and $\operatorname{cs}(G)=\left\{1, p, \ldots, p^{l}, p^{n}\right\}$; cf. [9, Lemma 2.2].

We show how to construct such a group $G$. Consider the $\mathbb{F}_{p}$-Lie algebra $\mathfrak{g}$, with $\mathbb{F}_{p}$-basis $\left(x_{1}, \ldots, x_{l}, \widetilde{x}_{1}, \ldots, \widetilde{x}_{l+n-1}, y_{1} \ldots, y_{n}\right)$, subject only to the relations:

$$
\left[x_{i}, \widetilde{x}_{j}\right]=\left\{\begin{array}{ll}
y_{j-i+1} & \text { if } i \leq j \leq i+n-1, \\
0 & \text { otherwise, }
\end{array} \quad \text { for } i \in[l], j \in[l+n-1] .\right.
$$

With respect to this basis the commutator matrix $B(\mathbf{Y}) \in \operatorname{Mat}\left(2 l+n-1, \mathbb{F}_{p}[\mathbf{Y}]\right)$ is

$$
\left(\begin{array}{cc}
0 & U(\mathbf{Y}) \\
-U(\mathbf{Y})^{\operatorname{tr}} & 0
\end{array}\right), \text { where } U(\mathbf{Y})=\left(\begin{array}{ccccccc}
Y_{1} & Y_{2} & \cdots & Y_{n} & & & \\
& Y_{1} & Y_{2} & \cdots & Y_{n} & & \\
& & \ddots & \ddots & & \ddots & \\
& & & Y_{1} & Y_{2} & \cdots & Y_{n}
\end{array}\right)
$$


The commutator matrix $A(\mathbf{X}) \in \operatorname{Mat}\left((2 l+n-1) \times n, \mathbb{F}_{p}[\mathbf{X}]\right)$ is

$$
\left(\begin{array}{cccc}
X_{l+1} & X_{l+2} & \cdots & X_{l+n} \\
X_{l+2} & X_{l+3} & \cdots & X_{l+n+1} \\
\vdots & & & \vdots \\
X_{2 l} & X_{2 l+1} & \cdots & X_{2 l+n-1} \\
-X_{1} & & & \\
\vdots & -X_{1} & & \\
-X_{l} & \vdots & \ddots & \\
& -X_{l} & & -X_{1} \\
& & \ddots & \vdots \\
& & & -X_{l}
\end{array}\right) .
$$

Note that $\left\{\operatorname{rk}(B(\mathbf{y})): \mathbf{y} \in \mathbb{F}_{p}^{n}\right\}=\{0, l\}$ and $\left\{\operatorname{rk}(A(\boldsymbol{x})): \boldsymbol{x} \in \mathbb{F}_{p}^{2 l+n-1}\right\}=\{0,1, \ldots, l, n\}$. Therefore $\operatorname{cd}(\exp (\mathfrak{g}))$ and $\operatorname{cs}(\exp (\mathfrak{g}))$ are as stated.

\section{Relatively fReE $p$-GRoups of exponent $p$}

Let $p$ be a prime and let $r \geq 2, c \geq 1$. Ito and Mann [19] study the numbers of classes and characters of the relatively free $p$-groups in the variety of groups of exponent $p$ and nilpotency class $c$ on $r$ generators. Our methods apply when $c<p$. We consider, more generally, the groups $F_{r, c}\left(\mathbb{F}_{q}\right):=\exp \left(\mathfrak{f}_{r, c}\left(\mathbb{F}_{q}\right)\right)$, where $q=p^{f}$ and $\mathfrak{f}_{r, c}\left(\mathbb{F}_{q}\right)$ is the free nilpotent $\mathbb{F}_{q}$-Lie algebra of class $c$ on $r$ generators. The orders of the terms of the lower central series of $\mathfrak{f}_{r, c}\left(\mathbb{F}_{q}\right)$, and so of $F_{r, c}\left(\mathbb{F}_{q}\right)$, may be expressed in terms of the Witt formula (2.1): for $i \in[c]$,

$$
\left|\gamma_{i}\left(\mathfrak{f}_{r, c}\left(\mathbb{F}_{q}\right)\right): \gamma_{i+1}\left(\mathfrak{f}_{r, c}\left(\mathbb{F}_{q}\right)\right)\right|=\left|\gamma_{i}\left(F_{r, c}\left(\mathbb{F}_{q}\right)\right): \gamma_{i+1}\left(F_{r, c}\left(\mathbb{F}_{q}\right)\right)\right|=q^{W_{r}(i)} ;
$$

cf. [19, Proposition 1]. We often write $F$ for $F_{r, c}\left(\mathbb{F}_{q}\right), \mathfrak{f}$ for $\mathfrak{f}_{r, c}\left(\mathbb{F}_{q}\right)$ and $W$ for $W_{r}$.

5.1. Proof of Theorem 2.6. We first prove a lemma about free Lie algebras over arbitrary fields. Let $\mathbb{K}$ be a field and $L$ a free $\mathbb{K}$-Lie algebra of rank at least 2 . We fix a Lie basis $\mathcal{B}$ for $L$ and, for $m \in \mathbb{N}$, define $L_{m}$ as the $\mathbb{K}$-linear span of $m$-fold Lie products of elements of $\mathcal{B}$. The standard grading $L=\bigoplus_{m=1}^{\infty} L_{m}$ determines the lower central series filtration $L^{i}:=\bigoplus_{j=i}^{\infty} L_{j}$ of $L$. By convention, $L^{i}:=L$ for $i \leq 0$.

Let $u=\sum_{i=1}^{\infty} u_{i} \in L$ be a nonzero element in the standard grading, i.e., $u_{i} \in L_{i}$ for all $i$. We denote by $\underline{u}$ the nonzero homogeneous component of lowest degree of $u$ in the standard grading: namely, $\underline{u}:=u_{\operatorname{deg}(u)}$, where $\operatorname{deg}(u):=\min \left\{i \in \mathbb{N}: u_{i} \neq 0\right\}$ is the degree of $u$.

Lemma 5.1. Let $u, v \in L$ with $u \neq 0$. If $[u, v] \in L^{i+1}$ for some positive integer $i$, then $v \in \mathbb{K} u+L^{i+1-\operatorname{deg} u}$.

Proof. Without loss of generality, assume $v \notin \mathbb{K} u$. By the Shirshov-Witt theorem [26. Theorem 2.5], every subalgebra of $L$ is free, and so $[u, v] \neq 0$. Now $[u, v]=$ $[\underline{u}, \underline{v}]+z$ for some $z \in L$ with $\operatorname{deg} z>\operatorname{deg} u+\operatorname{deg} v$.

If $[\underline{u}, \underline{v}] \neq 0$, then it is homogeneous of degree $\operatorname{deg} u+\operatorname{deg} v$. In this case $[u, v]=$ $[\underline{u}, \underline{v}]$ and so $[u, v] \notin L^{\operatorname{deg} u+\operatorname{deg} v+1}$. By hypothesis $[u, v] \in L^{i+1}$, so $i+1<\overline{\operatorname{deg} u}+$ $\operatorname{deg} v+1$ or, equivalently, $\operatorname{deg} v \geq i+1-\operatorname{deg} u$ which implies that $v \in L^{i+1-\operatorname{deg} u}$. 
If $[\underline{u}, \underline{v}]=0$, then the Shirshov-Witt theorem implies that $\underline{v}=k \underline{u}$ for some nonzero $k \in \mathbb{K}$. Thus $\operatorname{deg}(v-k \underline{u})>\operatorname{deg} u$. Since $\underline{v-k u} \notin \mathbb{K} \underline{u}$ and so $[\underline{u}, \underline{v}-k v] \neq 0$, we may apply the argument of the previous paragraph to $\underline{v-k u}$ instead of $\underline{v}$, deducing that $v-k u \in L^{i+1-\operatorname{deg} u}$.

The Lazard correspondence implies the following.

Lemma 5.2. Let $g \in F=F_{r, c}\left(\mathbb{F}_{q}\right)$ and $i \in[c]$. If $g \in \gamma_{i}(F) \backslash \gamma_{i+1}(F)$, then $C_{F}(g)=\left\langle g, \gamma_{c-i+1}(F)\right\rangle$.

We now prove Theorem 2.6. By Lemma 5.2, the conjugacy class sizes in $F=$ $F_{r, c}\left(\mathbb{F}_{q}\right)$ are the indices of the subgroups $\left\langle g, \gamma_{c-i+1}(F)\right\rangle$, for $g \in \gamma_{i}(F) \backslash \gamma_{i+1}(F)$ and $i \in[c]$. If $i \geq c-i+1$, then $\left\langle g, \gamma_{c-i+1}(F)\right\rangle=\gamma_{c-i+1}(F)$, which has index $q^{\sum_{j=1}^{c-i} W(j)}$ in $F$; see (5.1). If $i<c-i+1$, then $\left|\left\langle g, \gamma_{c-i+1}(F)\right\rangle: \gamma_{c-i+1}(F)\right|=q$, and so $\left|F:\left\langle g, \gamma_{c-i+1}(F)\right\rangle\right|=q^{-1+\sum_{j=1}^{c-i} W(j)}$. Thus if $g \in \gamma_{i}(F) \backslash \gamma_{i+1}(F)$, then the conjugacy class of $g$ has size $q^{k(r, c, i)}$.

The statement that $\operatorname{cc}_{0}(F)=q^{W(c)}$ follows immediately from (5.1), as $Z(F)=$ $\gamma_{c}(F)$. Note that $0=k(r, c, c)$. To determine $\operatorname{cc}_{j f}(F)$ where $j=k(r, c, i) \neq 0$, it suffices to count the elements in each $\gamma_{i}(F) \backslash \gamma_{i+1}(F)$ such that $k(r, c, i)=j$ and to observe that these elements fall into conjugacy classes of equal size $q^{j}$. Thus

$$
\begin{aligned}
\operatorname{cc}_{j f}(F) & =\sum_{\{i \in[c-1]: k(r, c, i)=j\}}\left|\gamma_{i}(F) \backslash \gamma_{i+1}(F)\right| q^{-j} \\
& =\sum_{\{i \in[c-1]: k(r, c, i)=j\}}\left(q^{W_{r}(i)}-1\right) q^{-j+\sum_{\ell=i+1}^{c} W_{r}(\ell)} .
\end{aligned}
$$

This concludes the proof of Theorem 2.6.

Corollary 5.3. Let $r \geq 2, c \geq 1$ and $q$ be a power of $p>c$. The entries of the class vector $\operatorname{cc}\left(F_{r, c}\left(\mathbb{F}_{q}\right)\right)$, and hence also the class number $k\left(F_{r, c}\left(\mathbb{F}_{q}\right)\right)$, are given by a polynomial in $q$ which depends only on $r$ and $c$. Expanded in $q-1$, this polynomial has nonnegative coefficients.

Proof. Theorem 2.6 shows that the relevant quantities may be written as sums of terms of the form $q^{\alpha}$ and $\left(q^{\beta}-1\right) q^{\gamma}$ for nonnegative integers $\alpha, \beta, \gamma$.

Remark 5.4. Corollary 5.3 may be compared to an analogous conjecture about the class vectors of the groups $U_{n}\left(\mathbb{F}_{q}\right)$ of upper-unitriangular matrices over $\mathbb{F}_{q} ;$ cf. [30]. Isaacs [18] formulates a similar conjecture for the character vectors of these groups; it is proved in 8 . for $n \leq 13$. In Remark 5.12 we note that the corresponding statement for the character vectors of the groups $F_{2,5}\left(\mathbb{F}_{q}\right)$ is false.

5.2. Proof of Theorem 2.5. We recall the well-known definition of a Hall basis; cf. [21].

Definition 5.5. Let $\Delta=\left\{e_{1}^{(1)}, \ldots, e_{r}^{(1)}\right\}$ be a set of Lie algebra generators for $\mathfrak{f}=$ $\mathfrak{f}_{r, c}\left(\mathbb{F}_{q}\right)$. If $u \in \mathfrak{f}$ is a Lie product of elements from $\Delta$, then $u$ has weight $\operatorname{wt}(u)=i$ if $u \in \gamma_{i}(\mathfrak{f}) \backslash \gamma_{i+1}(\mathfrak{f})$. A Hall basis (on $\Delta$ ) for $\mathfrak{f}$ is a well-ordered subset $\mathcal{H}$ of $\mathfrak{f}$, satisfying the following:

(1) $\Delta \subseteq \mathcal{H}$.

(2) If $u, v \in \mathcal{H}$, then $[u, v] \in \mathcal{H}$ if and only if

$$
u>v \text { and }\left(u=\left[u_{1}, u_{2}\right] \text { implies } u_{2} \leq v\right) .
$$


(3) If $w \in \mathcal{H} \backslash \Delta$, then $w=[u, v]$ for some $u, v \in \mathcal{H}$ satisfying (5.2).

(4) If $u, v \in \mathcal{H}$ and $\operatorname{wt}(u)>\operatorname{wt}(v)$, then $u>v$.

Elements of $\mathcal{H}$ are basic commutators. For $i \in[c]$, we set $\mathcal{H}^{(i)}:=\{h \in \mathcal{H}: \operatorname{wt}(h)=$ $i\}$ and label the basic commutators of weight $i$ so that $\mathcal{H}^{(i)}=\left\{e_{1}^{(i)}, \ldots, e_{W(i)}^{(i)}\right\}_{<}$. Observe that $g^{(i)}:=\left[e_{2}^{(1)},{ }_{i-1} e_{1}^{(1)}\right] \in \mathcal{H}^{(i)}$.

Choose a Hall basis $\mathcal{H}$ for $\mathfrak{f}$. It is well known that the elements of $\bigcup_{i=2}^{c} \mathcal{H}^{(i)}$ yield an $\mathbb{F}_{q}$-basis for the derived Lie algebra $\mathfrak{f}^{\prime}$, and that the residue classes of the elements of $\bigcup_{i=1}^{c-1} \mathcal{H}^{(i)}$ yield an $\mathbb{F}_{q}$-basis for the cocentre $\mathfrak{f} / Z(\mathfrak{f})$. Observe that the commutator matrix $B(\mathbf{Y}) \in \operatorname{Mat}\left(\sum_{j=1}^{c-1} W(j), \mathbb{F}_{q}[\mathbf{Y}]\right)$ with respect to $\mathcal{H}$ is a skew-symmetric matrix of $\mathbb{Z}$-linear forms in $b=\sum_{j=2}^{c} W(j)$ variables. We label the variables as follows. For $k \in[2, c]$ we write $\mathbf{Y}^{(k)}=\left(Y_{1}^{(k)}, \ldots, Y_{W(k)}^{(k)}\right)$. Thus $\mathbf{Y}=\left(\mathbf{Y}^{(k)}\right)_{k \in[2, c]}$ and

$$
B(\mathbf{Y})=\left(\begin{array}{cccc}
B_{1,1}\left(\mathbf{Y}^{(2)}\right) & B_{1,2}\left(\mathbf{Y}^{(3)}\right) & \ldots & B_{1, c-1}\left(\mathbf{Y}^{(c)}\right) \\
B_{2,1}\left(\mathbf{Y}^{(3)}\right) & B_{2,2}\left(\mathbf{Y}^{(4)}\right) & . \cdot & 0 \\
\vdots & . \cdot & 0 & \\
B_{c-1,1}\left(\mathbf{Y}^{(c)}\right) & 0 & &
\end{array}\right)
$$

where $B_{i, j}\left(\mathbf{Y}^{(i+j)}\right)$ is the zero matrix if $i+j>c$, and for $i, j \in[c-1]$,

$$
B_{i, j}:=B_{i, j}\left(\mathbf{Y}^{(i+j)}\right)=-B_{j, i}\left(\mathbf{Y}^{(i+j)}\right)^{\operatorname{tr}} \in \operatorname{Mat}\left(W(i) \times W(j), \mathbb{F}_{q}\left[\mathbf{Y}^{(i+j)}\right]\right) .
$$

For each $k \in[2, c]$, the variables $\mathbf{Y}^{(k)}$ only occur in the matrices $B_{i, j}$ with $i+j=k$. It follows from [28, Theorem 1] that

- if $j>i$ and $i$ does not divide $j$, then $B_{i, j}$ is generic: there are no linear relations among its entries;

- if $i=j$, then $B_{i, i}$ is generic skew-symmetric: the only linear relations between its entries are those resulting from the identity $B_{i, i}=-B_{i, i}^{\operatorname{tr}}$.

To prove Theorem 2.5 it suffices, by Theorem B, to show that

$$
\left\{\operatorname{rk}(B(\mathbf{y})): \mathbf{y} \in \mathbb{F}_{q}^{b}\right\}=2[n(r, c)]_{0} .
$$

The containment $\subseteq$ in (5.4) is clear, as the rank of $B(\mathbf{y})$ is clearly bounded from above by $2 n(r, c)$. We establish the containment $\supseteq$ in (5.4) by induction on $c$. For $c=1$ there is nothing to prove, and the case $c=2$ is covered by Proposition 2.4, so let $c>2$. The induction step is divided into five steps. To ensure that the induction hypothesis is applicable, we assume further that $(r, c) \neq(2,4)$. The statement of Theorem 2.5 for groups of the form $F_{2,4}\left(\mathbb{F}_{q}\right)$ follows from Proposition 5.10.

Step 1 . By the induction hypothesis, we can obtain every rank in $2[n(r, c-1)]_{0}$ by setting the 'new' variables $\mathbf{Y}^{(c)}$ to zero, and arguing as for $c-1$.

Step 2. Let $\rho \in 2[n(r, c-1)+1, n(r, c)]$. If there exists a vector $\mathbf{y}=\left(\mathbf{y}^{(k)}\right)_{k \in[2, c]} \in$ $\mathbb{F}_{q}^{b}$, with $\mathbf{y}^{(k)}=\mathbf{0}$ for $k<c$, satisfying

(1) $\operatorname{rk}\left(B_{c-i, i}\left(\mathbf{y}^{(c)}\right)\right)=\min \{W(i), W(c-i)\}=W(i)$ for $i<m=\lfloor c / 2\rfloor$,

(2) $\operatorname{rk}\left(B_{c-m, m}\left(\mathbf{y}^{(c)}\right)\right)= \begin{cases}\rho / 2-\sum_{i=1}^{m-1} W(i) & \text { if } c=2 m+1, \\ \rho-2 \sum_{i=1}^{m-1} W(i) & \text { if } c=2 m,\end{cases}$ 
then $\operatorname{rk}(B(\mathbf{y}))=\rho$. Indeed, $B(\mathbf{y})$ is a matrix with nonzero blocks $B_{i, j}\left(\mathbf{y}^{(c)}\right)$ only in the positions $(i, j)$ where $i+j=c$. Moreover, apart from the 'central block' $B_{m, m}\left(\mathbf{y}^{(c)}\right)$ if $c=2 m$, or 'central blocks' $B_{m, m+1}\left(\mathbf{y}^{(c)}\right)$ and $B_{m+1, m}\left(\mathbf{y}^{(c)}\right)$ if $c=$ $2 m+1$, all blocks have maximal rank.

Step 3. We now prove that such a vector y exists. As $(r, c) \neq(2,3), W(i)<W(j)$ whenever $i<j$. It suffices to show that, for each $i<c / 2$, the matrix $B_{c-i, i}$ has a square submatrix

$$
\widetilde{B}_{c-i, i}:=\widetilde{B}_{c-i, i}\left(\mathbf{Y}^{(c)}\right) \in \operatorname{Mat}\left(W(i), \mathbb{F}_{q}\left[\mathbf{Y}^{(c)}\right]\right),
$$

obtained by choosing $W(i)$ suitable rows of $B_{c-i, i}$, with the property that there are no linear relations among the entries $\left(\widetilde{B}_{c-i, i}\right)_{s t}, 1 \leq s \leq t \leq W(i)$, for $1<i<c / 2$, and, if $c=2 m$, the entries $\left(B_{m, m}\right)_{s t}, 1 \leq s \leq t \leq W(m)$. Indeed, given such matrices $\widetilde{B}_{c-i, i}$, it is easy to construct a vector $\mathbf{y}^{(c)}$ such that, for all $i<m$, $\widetilde{B}_{c-i, i}\left(\mathbf{y}^{(c)}\right)$ is lower-unitriangular (and thus, in particular, of maximal rank $W(i)$ ) and the central blocks have the required ranks: namely, we set the diagonal entries of $\widetilde{B}_{i, c-i}$ equal to one, and all the $(s, t)$-entries of $\widetilde{B}_{i, c-i}$ for $s<t$ equal to zero.

If $c=2 m$, the matrix $B_{m, m}$ is generic skew-symmetric, by [28, Theorem 1], and so attains every rank in $2[\lfloor W(m) / 2\rfloor]_{0}$.

Step 4. For $i<c / 2$ we now exhibit such a submatrix $\widetilde{B}_{c-i, i}$ of $B_{c-i, i}$. By definition of the commutator matrix $B$, the matrix $B_{c-i, i}$ is defined by $\left(B_{c-i, i}\right)_{s t}=$ $\sum_{k=1}^{W(c)} \lambda_{s t}^{k} Y_{k}^{(c)}$, where $\left[e_{s}^{(c-i)}, e_{t}^{(i)}\right]=\sum_{k=1}^{W(c)} \lambda_{s t}^{k} e_{k}^{(c)}$, where $s \in W(c-i), t \in W(i)$. It suffices to find $S=\left\{s_{1}, \ldots, s_{W(i)}\right\}<\subseteq[W(c-i)]$, indexing $W(i)$ rows of $B_{c-i, i}$, such that

$$
\left[e_{s_{l}}^{(c-i)}, e_{t}^{(i)}\right] \in \mathcal{H}^{(c)} \text { for } l \in[W(i)] \text { and } t \in[l, W(i)] .
$$

We then set $\widetilde{B}_{c-i, i}:=\left(\left(B_{c-i, i}\right)_{s t}\right)_{s \in S, t \in[W(i)]}$.

Step 5 . To find such a subset $S$ of $[W(c-i)]$, we distinguish three cases.

Case $(i): i>(c-i) / 2$. Every pair $\left(e_{s}^{(c-i)}, e_{t}^{(i)}\right) \in \mathcal{H}^{(c-i)} \times \mathcal{H}^{(i)}$ has the property that $\left[e_{s}^{(c-i)}, e_{t}^{(i)}\right] \in \mathcal{H}^{(c)}$. Indeed, since $i<c / 2$, we deduce that $i<c-i$, so $e_{t}^{(i)}<e_{s}^{(c-i)}$. If $e_{s}^{(c-i)}=\left[u_{1}, u_{2}\right]$ for some $u_{1}, u_{2} \in \mathcal{H}$, then $\operatorname{wt}\left(u_{2}\right) \leq(c-i) / 2<i$ by (5.2), so $u_{2}<e_{t}^{(i)}$, and hence $\left[e_{s}^{(c-i)}, e_{t}^{(i)}\right] \in \mathcal{H}^{(c)}$. Thus every $W(i)$-element subset $S$ of $[W(c-i)]$ satisfies (5.5).

Case $(i i): i<(c-i) / 2$. Let $t \in[W(i)]$. Since $i<c-2 i$, clearly $e_{s}^{(c-i)}:=$ $\left[g^{(c-2 i)}, e_{t}^{(i)}\right] \in \mathcal{H}^{(c-i)}$; so, if $v \geq t$, then $\left[e_{s}^{(c-i)}, e_{v}^{(i)}\right] \in \mathcal{H}^{(c)}$. Therefore the set $S$ of indices of the $W(i)$ elements $\left[g^{(c-2 i)}, e_{t}^{(i)}\right]$, for $t \in[W(i)]$, satisfies (5.5).

Case (iii): $i=(c-i) / 2$. Let $t \in[W(i)]$. If $t<W(i)$, then we set $e_{s}^{(2 i)}:=$ $\left[e_{W(i)}^{(i)}, e_{t}^{(i)}\right] \in \mathcal{H}^{(2 i)}$ and observe that $\left[e_{s}^{(2 i)}, e_{v}^{(i)}\right] \in \mathcal{H}^{(c)}$ for all $v \geq t$. If $t=W(i)$, then $\left[g^{(2 i)}, e_{W(i)}^{(i)}\right] \in \mathcal{H}^{(c)}$. The set of indices of the $W(i)$ elements $g^{(2 i)}$ and $\left[e_{W(i)}^{(i)}, e_{t}^{(i)}\right]$, for $t \in[W(i)-1]$, satisfies (5.5).

This concludes the proof of Theorem 2.5 . 
5.3. Taketa bounds for $F_{r, c}\left(\mathbb{F}_{q}\right)$. The Taketa problem asks for a bound to the derived length $\operatorname{dl}(G)$ of a finite solvable group $G$ in terms of the number of its character degrees; see, for example, 22. It is known that $\operatorname{dl}(G)$ is bounded by a linear function in $|\operatorname{cd}(G)|$. Isaacs conjectured that the bound for $p$-groups is logarithmic. It cannot be better than logarithmic, as the family $\left(U_{n}\left(\mathbb{F}_{q}\right)\right)$ shows: $\operatorname{dl}\left(U_{n}\left(\mathbb{F}_{q}\right)\right)=\left\lceil\log _{2}(n)\right\rceil$, but $\left|\operatorname{cd}\left(U_{n}\left(\mathbb{F}_{q}\right)\right)\right| \sim n^{2} / 4$; cf. [16].

Our results exhibit double-logarithmic Taketa bounds for the groups $F_{r, c}\left(\mathbb{F}_{q}\right)$, a family of groups of unbounded derived length. Indeed, $\operatorname{dl}\left(F_{r, c}\left(\mathbb{F}_{q}\right)\right)=\left\lceil\log _{2}(c)\right\rceil$, whereas $n(r, c) \sim r^{\lfloor c / 2\rfloor}$. Thus $\operatorname{dl}\left(F_{r, c}\left(\mathbb{F}_{q}\right)\right) \leq c_{1} \log \log \left(\left|\operatorname{cd}\left(F_{r, c}\left(\mathbb{F}_{q}\right)\right)\right|\right)+c_{2}$ for suitable constants $c_{1}, c_{2}$.

We also observe that there is a logarithmic bound to the derived length of the groups $F_{r, c}\left(\mathbb{F}_{q}\right)$ in terms of their numbers of class sizes. In fact, $\left|\operatorname{cs}\left(F_{r, c}\left(\mathbb{F}_{q}\right)\right)\right|=c+1$ (unless both $r$ and $c$ are very small), so $\operatorname{dl}\left(F_{r, c}\left(\mathbb{F}_{q}\right)\right) \leq c_{3} \log \left(\left|\operatorname{cs}\left(F_{r, c}\left(\mathbb{F}_{q}\right)\right)\right|\right)$. The $\left(\log _{q}\right.$ of the $)$ class sizes of the groups $U_{n}\left(\mathbb{F}_{q}\right)$ form an interval of length $\left(\begin{array}{c}n-1 \\ 2\end{array}\right)$ (cf. [29]), also yielding a logarithmic bound for this family.

5.4. Numbers of characters. Theorem 2.5 describes the support of the character $\operatorname{vectors} \operatorname{ch}\left(F_{r, c}\left(\mathbb{F}_{q}\right)\right)$, showing that the numbers $\operatorname{ch}_{i f}\left(F_{r, c}\left(\mathbb{F}_{q}\right)\right)$, for $i \in[n(r, c)]_{0}$, are nonzero. We make one observation on the order of magnitude of the number of characters of maximal degree $q^{n(r, c)}$. Define

$$
N(r, c):=\sum_{i=1}^{c} W_{r}(i)-2 n(r, c) .
$$

Lemma 5.6. Let $(r, c) \neq(2,3), p>c$ and $q=p^{f}$. Then

$$
\lim _{q \rightarrow \infty} \frac{\operatorname{ch}_{n(r, c) f}\left(F_{r, c}\left(\mathbb{F}_{q}\right)\right)}{q^{N(r, c)}}=1 .
$$

Proof. The Lang-Weil estimate (cf. 24]) for the number of rational points on varieties over finite fields implies that $\operatorname{ch}_{n(r, c) f}\left(F_{r, c}\left(\mathbb{F}_{q}\right)\right) \sim q^{r-2 n(r, c)}\left|F_{r, c}\left(\mathbb{F}_{q}\right)^{\prime}\right|=$ $q^{N(r, c)}$.

By Theorem 2.5, the smallest degree of a nonlinear character of $F_{r, c}\left(\mathbb{F}_{q}\right)$ is $q=p^{f}$. We now count the number of characters of $F_{r, c}\left(\mathbb{F}_{q}\right)$ having degree $q=p^{f}$, so generalizing [19, Theorem 7].

Proposition 5.7. Let $r \geq 2, p>c>2$ and $q=p^{f}$. Then

$$
\operatorname{ch}_{f}\left(F_{r, c}\left(\mathbb{F}_{q}\right)\right)=\frac{q^{r-2}\left(q^{r}-1\right)\left(q^{(r-1)(c-1)+1}+q^{(r-1)(c-1)}-q^{r}-1\right)}{q^{2}-1} .
$$

Proof. Let $B(\mathbf{Y})$ be the commutator matrix with respect to a Hall basis for $\mathfrak{f}_{r, c}\left(\mathbb{F}_{q}\right)$, and recall the definition (5.3) of the matrices $B_{i, j}$. For $\mathbf{y} \in \mathbb{F}_{q}^{b}$ we define

$$
u(\mathbf{y}):=\max \left\{i \in[2, c]: \text { there exists } j \in[i-1] \text { such that } B_{j, i-j}(\mathbf{y}) \neq 0\right\} .
$$

For $s \in[2, c]$, we now compute the quantity $n_{s}:=\#\left\{\mathbf{y} \in \mathbb{F}_{q}^{b}: u(\mathbf{y})=s, \operatorname{rk}(B(\mathbf{y}))=\right.$ $2\}$. This suffices, $\operatorname{as~}_{\operatorname{ch}}\left(F_{r, c}\left(\mathbb{F}_{q}\right)\right)=q^{r-2} \nu_{f}\left(F_{r, c}\left(\mathbb{F}_{q}\right)\right)=q^{r-2} \sum_{s=2}^{c} n_{s}$.

For $s=2$, Proposition 2.4 for $k=1$ implies that $n_{2}=\left(q^{r}-1\right)\left(q^{r-1}-1\right) /\left(q^{2}-1\right)$. For $s>2$, we claim that

$$
n_{s}=\frac{q^{(r-1)(s-2)}\left(q^{r}-1\right)\left(q^{r-1}-1\right)}{(q-1)} .
$$


First note that if $u(\mathbf{y})=s$ and $\operatorname{rk}(B(\mathbf{y}))=2$, then $\operatorname{rk}\left(B_{1, s-1}(\mathbf{y})\right)=1$ and $B_{i, s-i}(\mathbf{y})=0$ for $i \in[2, s-2]$; see, for example, [28, Theorem 1]. In fact, after a suitable change of basis for $\mathfrak{f}_{r, c}\left(\mathbb{F}_{q}\right)$, we may assume that $B(\mathbf{y})$ has zero entries everywhere except the first row and column. We claim that

$$
\begin{aligned}
\#\{\mathbf{y} & \left.\in \mathbb{F}_{q}^{W(s)}: \operatorname{rk}\left(B_{1, s-1}(\mathbf{y})\right)=1, B_{i, s-i}(\mathbf{y})=0 \text { for } i \in[2, s-2]\right\} \\
& =\frac{\left(q^{r}-1\right)\left(q^{r-1}-1\right)}{(q-1)} .
\end{aligned}
$$

Indeed, there are $q^{r-1}$ ways to fill in a row of $B_{1, s-1}(\mathbf{Y})$ so that all other rows are zero. To see this, assume without loss of generality that this is the first row, and note that exactly $r-1$ of the Lie products of the form $\left[e_{i}^{(s-1)}, e_{1}\right]$, where $e_{i}^{(s-1)}$ is a basic commutator of weight $s-1$, are basic, namely the ones of the form $\left[e_{i}, s-2 e_{1}\right]$ where $i \in[2, r]$. All other Lie products of the form $\left[e_{i}^{(s-1)}, e_{1}\right]$ are linear combinations of other basic commutators of weight $s$. The variables associated to these occur in some other row of $B_{1, s-1}(\mathbf{Y})$, or in some $B_{i, s-i}(\mathbf{Y})$ for $i \geq 2$, and so have the value zero. Up to nonzero scalars, there are thus $\left(q^{r-1}-1\right) /(q-1)$ ways to fill a row without obtaining a zero row. Every row of $B_{1, s-1}(\mathbf{Y})$ is a linear multiple of such a nonzero row, and only one of the $q^{r}$ possibilities yields the zero matrix. This establishes the claim.

We also claim that, for each $\mathbf{y} \in \mathbb{F}_{q}^{W(s)}$ such that $\operatorname{rk}\left(B_{1, s-1}(\mathbf{y})\right)=1$ and $B_{i, s-i}(\mathbf{y})=0$ for $i \in[2, s-2]$, there are $q^{(r-1)(s-2)}$ ways to choose $\mathbf{y}^{\prime} \in \mathbb{F}_{q}^{b}$ such that $\operatorname{rk}\left(B\left(\mathbf{y}^{\prime}, \mathbf{y}\right)\right)=1$. Indeed, again without loss we may assume that $B_{1, s-1}(\mathbf{y})$ is supported only on its first row. By the arguments in the previous paragraph, each of the matrices $B_{1, i}(\mathbf{Y})$, for $i \in[s-2]$, has exactly $r-1$ variables corresponding to basic commutators in its first row. All other entries in the first row are linear combinations of variables corresponding to basic commutators occurring in other rows. This establishes the claim, and so (5.6).

Summing over $s=2, \ldots, c$ establishes the result.

5.5. Results on $F_{r, c}\left(\mathbb{F}_{q}\right)$ for specific values of $r$ and $c$. We start with a lemma generalizing the opening remarks of [19, Section 3], thus dealing with the exceptional parameter values in Theorem 2.5 .

Proposition 5.8. Let $p \geq 5$ and let $q=p^{f}$.

$$
\operatorname{ch}\left(F_{2,3}\left(\mathbb{F}_{q}\right)\right)=\left(q^{2}, q^{3}-1\right)_{f} .
$$

Proof. We note that $W_{2}(1)=2, W_{2}(2)=1$ and $W_{2}(3)=2$. With respect to the Hall basis $\left\{e_{1}^{(1)}, e_{2}^{(1)}, g^{(2)},\left[g^{(2)}, e_{1}^{(1)}\right],\left[g^{(2)}, e_{2}^{(1)}\right]\right\}_{<}$for $\mathfrak{f}_{2,3}\left(\mathbb{F}_{q}\right)$ the commutator matrix

$$
B(\mathbf{Y})=\left(\begin{array}{cc|c} 
& -Y_{1} & -Y_{2} \\
Y_{1} & & -Y_{3} \\
\hline Y_{2} & Y_{3} &
\end{array}\right) .
$$

The claim follows immediately from Theorem B.

Proposition 5.9. Let $p \geq 5$ and let $q=p^{f}$.

$\operatorname{ch}\left(F_{3,3}\left(\mathbb{F}_{q}\right)\right)=\left(q^{3}, q\left(q^{3}-1\right)\left(q^{3}+q^{2}+1\right), q\left(q^{3}-1\right)\left(q^{5}+q^{4}-1\right), q^{4}(q-1)\left(q^{3}-q-1\right)\right)_{f}$. 
Proof. Set $F=F_{3,3}\left(\mathbb{F}_{q}\right)$. We note that $W_{3}(1)=3, W_{3}(2)=3$ and $W_{3}(3)=8$. With respect to a Hall basis for $\mathfrak{f}_{3,3}\left(\mathbb{F}_{q}\right)$, the commutator matrix $B(\mathbf{Y})$ is

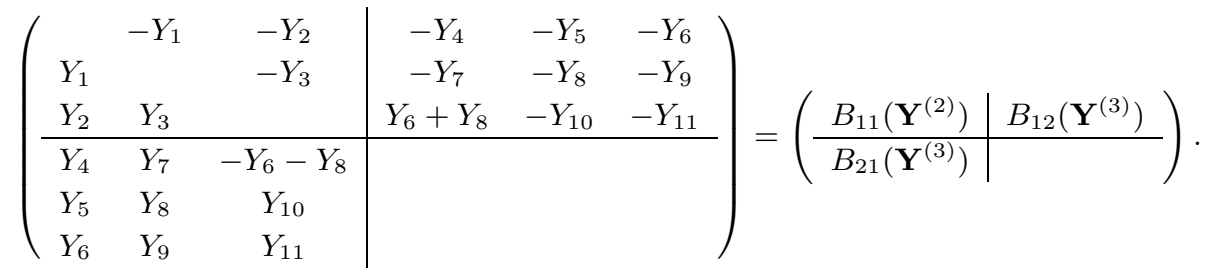

It suffices to prove our claim for $\operatorname{ch}_{2 f}(F)$. Indeed, $n(3,3)=3$, the claim for $\operatorname{ch}_{0}(F)$ is trivial, and that for $\operatorname{ch}_{f}(F)$ follows from Proposition 5.7. Furthermore, the class number $k(F)=\sum_{i=0}^{3} q^{3-2 i} \nu_{i f}(F)$ is $q^{9}-2 q^{8}-q^{6}-q^{5}$ by Theorem 2.6.

We claim that there are $q\left(q^{3}-1\right)\left(q^{5}+q^{4}-1\right)$ vectors $\mathbf{y} \in \mathbb{F}_{q}^{11}$ such that $\operatorname{rk}(B(\mathbf{y}))=$ 4. For such $\mathbf{y}$ we distinguish whether $\operatorname{rk}\left(B_{12}(\mathbf{y})\right)=1$ or $\operatorname{rk}\left(B_{12}(\mathbf{y})\right)=2$. In the former case, by (5.4) in the proof of Proposition 5.7, there are $(q+1)\left(q^{3}-1\right)$ vectors $\left(y_{4}, \ldots, y_{11}\right) \in \mathbb{F}_{q}^{8}$ yielding $\operatorname{rk}\left(B_{12}\left(y_{4}, \ldots, y_{11}\right)\right)=1$, and for each of these there are $q^{3}-q^{2}$ vectors $\left(y_{1}, y_{2}, y_{3}\right) \in \mathbb{F}_{q}^{3}$ such that $\operatorname{rk}\left(B\left(y_{1}, \ldots, y_{11}\right)\right)=4$. Thus

$$
\#\left\{\mathbf{y} \in \mathbb{F}_{q}^{11}: \operatorname{rk}(B(\mathbf{y}))=4, \operatorname{rk}\left(B_{12}(\mathbf{y})\right)=1\right\}=q^{2}\left(q^{2}-1\right)\left(q^{3}-1\right) .
$$

On the other hand, the set $N:=\left\{\mathbf{y} \in \mathbb{F}_{q}^{8}: \operatorname{rk}\left(B_{12}(\mathbf{y})\right)=2\right\}$ has cardinality $q\left(q^{3}-1\right)\left(q^{3}+q^{2}-1\right)$; cf. [2]. As every vector in $N$ gives rise to $q^{3}$ matrices $B(\mathbf{y})$ of rank 4 ,

$$
\#\left\{\mathbf{y} \in \mathbb{F}_{q}^{11}: \operatorname{rk}(B(\mathbf{y}))=4, \operatorname{rk}\left(B_{12}(\mathbf{y})\right)=2\right\}=q^{4}\left(q^{3}-1\right)\left(q^{3}+q^{2}-1\right)
$$

and thus

$$
\nu_{2 f}(F)=q^{2}\left(q^{2}-1\right)\left(q^{3}-1\right)+q^{4}\left(q^{3}-1\right)\left(q^{3}+q^{2}-1\right)=q^{2}\left(q^{3}-1\right)\left(q^{5}+q^{4}-1\right),
$$

which yields the claimed quantity for $\operatorname{ch}_{2 f}(F)=q^{3-4} \nu_{2 f}(F)$.

We obtain the following generalization of [19, Lemma 14].

Proposition 5.10. Let $p \geq 5$ and let $q=p^{f}$.

$$
\operatorname{ch}\left(F_{2,4}\left(\mathbb{F}_{q}\right)\right)=\left(q^{2}, q^{4}+q^{3}-q^{2}-1, q^{4}-q^{2}-q+1\right)_{f} .
$$

Proof. Note that $n(2,4)=2$. The formula for $\operatorname{ch}_{f}\left(F_{2,4}\left(\mathbb{F}_{q}\right)\right)$ is given by Proposition 5.7 and the class number $k\left(F_{2,4}\left(\mathbb{F}_{q}\right)\right)$ is given by Theorem 2.6.

Proposition 5.11. Let $p \geq 7$ and let $q=p^{f}$. The nonzero values of $\operatorname{ch}_{i}\left(F_{2,5}\left(\mathbb{F}_{q}\right)\right)$ are given as follows:

$$
\begin{array}{r|l}
i & \operatorname{ch}_{i}\left(F_{2,5}\left(\mathbb{F}_{q}\right)\right) \\
\hline 0 & q^{2} \\
f & (q-1)\left(q^{4}+2 q^{3}+2 q^{2}+q+1\right) \\
2 f & (q-1)\left(q^{7}+2 q^{6}+3 q^{5}+2 q^{4}+q^{3}-q-1\right) \\
3 f & q^{2}\left(q^{2}-1\right)\left(q^{4}-q-1\right)
\end{array}
$$

Proof. Set $F=F_{2,5}\left(\mathbb{F}_{q}\right)$, and note that $n(2,5)=3$. The statement about $\operatorname{ch}_{0}(F)$ is trivial; the claim about $\operatorname{ch}_{f}(F)$ is a special case of Proposition 5.7. By Theorem 2.6. $k(F)=2 q^{8}+q^{7}-q^{5}-q^{4}$, so it suffices to compute, for instance, $\operatorname{ch}_{3 f}(F)$.

We now describe a Hall basis for $\mathfrak{f}_{2,5}\left(\mathbb{F}_{q}\right)$. We choose Lie generators $x$ and $y$, where $y<x$, and omit Lie brackets in left-normed Lie products, so, for example, 
$[[x, y], x]$ is represented by $x y x$. It is easily verified that the following elements form a Hall basis:

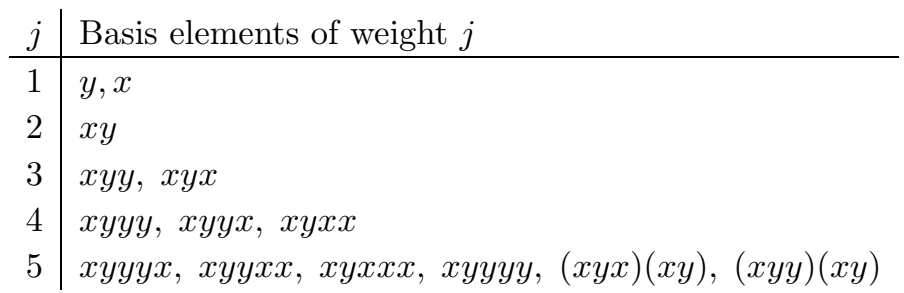

With respect to this basis, the commutator matrix

$B(\mathbf{Y})=\left(\begin{array}{cc|c|cc|ccc} & -Y_{1} & -Y_{2} & -Y_{4} & -Y_{5} & -Y_{10} & -Y_{11}-Y_{7} & -Y_{12}-Y_{8} \\ Y_{1} & & -Y_{3} & -Y_{5} & -Y_{6} & -Y_{7} & -Y_{8} & -Y_{9} \\ \hline Y_{2} & Y_{3} & & -Y_{11} & -Y_{12} & & & \\ \hline Y_{4} & Y_{5} & Y_{11} & & & & & \\ Y_{5} & Y_{6} & Y_{12} & & & & & \\ \hline Y_{10} & Y_{7} & & & & & & \\ Y_{11}+Y_{7} & Y_{8} & & & & & & \\ Y_{12}+Y_{8} & Y_{9} & & & & & & \end{array}\right)$.

It suffices to prove that $\nu_{3 f}(F)=q^{6}\left(q^{2}-1\right)\left(q^{4}-q-1\right)$. If $\mathbf{y} \in \mathbb{F}_{q}^{12}$ and $\operatorname{rk}(B(\mathbf{y}))=$ 6 , then $\left(y_{11}, y_{12}\right) \neq\{\mathbf{0}\}$. Fix $\left(y_{11}, y_{12}\right) \in \mathbb{F}_{q}^{2} \backslash\{\mathbf{0}\}$. It is easily checked that

$$
\#\left\{\mathbf{y}=\left(y_{7}, \ldots, y_{10}\right) \in \mathbb{F}_{q}^{4}: \operatorname{rk}\left(B_{14}(\mathbf{y})\right)=1\right\}=q(q+1) .
$$

Given $\mathbf{y}=\left(y_{7}, \ldots, y_{10}\right) \in \mathbb{F}_{q}^{4}$ with $\operatorname{rk}\left(B_{14}(\mathbf{y})\right)=1$, there are $q^{5}(q-1)$ ways to choose $\left(y_{1}, \ldots, y_{6}\right) \in \mathbb{F}_{q}^{6}$ such that $\operatorname{rk}\left(B\left(y_{1}, \ldots, y_{12}\right)\right)=6$. Similarly,

$$
\#\left\{\mathbf{y}=\left(y_{7}, \ldots, y_{10}\right) \in \mathbb{F}_{q}^{4}: \operatorname{rk}\left(B_{14}(\mathbf{y})\right)=2\right\}=q^{4}-q(q+1) .
$$

Given $\mathbf{y}=\left(y_{7}, \ldots, y_{10}\right) \in \mathbb{F}_{q}^{4}$ with $\operatorname{rk}\left(B_{14}(\mathbf{y})\right)=2$, there are $q^{6}$ ways to choose $\left(y_{1}, \ldots, y_{6}\right) \in \mathbb{F}_{q}^{6}$ such that $\operatorname{rk}\left(B\left(y_{1}, \ldots, y_{12}\right)\right)=6$. Thus

$\nu_{3 f}(F)=\left(q^{2}-1\right)\left(q^{5}(q-1) \cdot q(q+1)+q^{6} \cdot\left(q^{4}-\left(q^{2}+q\right)\right)\right)=q^{6}\left(q^{2}-1\right)\left(q^{4}-q-1\right)$

as claimed.

Remark 5.12. We note that $\operatorname{ch}_{3 f}\left(F_{2,5}\left(\mathbb{F}_{q}\right)\right)$ is given by a polynomial in $q$ and its expansion in $v:=q-1$ has both positive and negative coefficients. Indeed

$$
\operatorname{ch}_{3 f}\left(F_{2,5}\left(\mathbb{F}_{q}\right)\right)=v(v+2)(v+1)^{2}\left(v^{4}+4 v^{3}+6 q^{2}+3 v-1\right) .
$$

We observe this phenomenon only for the family of groups $F_{2,5}\left(\mathbb{F}_{q}\right)$, for $p \geq 7$; in all other families we considered the corresponding coefficients are nonnegative.

\section{ACKNOWLEDGEMENTS}

The authors acknowledge support from the Alexander von Humboldt Foundation, the EPSRC, the Marsden Fund of New Zealand and the Royal Society. The authors also thank I. M. Isaacs, L. G. Kovács and Avinoam Mann for helpful discussions. 


\section{REFERENCES}

[1] Nir Avni, Benjamin Klopsch, Uri Onn, and Christopher Voll, Representation zeta functions of compact p-adic analytic groups and arithmetic groups, Duke Math. J. 162 (2013), no. 1, 111-197, DOI 10.1215/00127094-1959198. MR3011874

[2] Edward A. Bender, On Buckheister's enumeration of $n \times n$ matrices, J. Combinatorial Theory Ser. A 17 (1974), 273-274. MR0347622(50 \#125)

[3] Nigel Boston and I. M. Isaacs, Class numbers of p-groups of a given order, J. Algebra 279 (2004), no. 2, 810-819, DOI 10.1016/j.jalgebra.2004.03.006. MR2078943 (2005f:20034)

[4] Mitya Boyarchenko, Representations of unipotent groups over local fields and Gutkin's conjecture, Math. Res. Lett. 18 (2011), no. 3, 539-557, DOI 10.4310/MRL.2011.v18.n3.a14. MR2802587(2012d:22021)

[5] Mitya Boyarchenko and Maria Sabitova, The orbit method for profinite groups and a p-adic analogue of Brown's theorem, Israel J. Math. 165 (2008), 67-91, DOI 10.1007/s11856-0081004-3. MR 2403615 (2009b:20046)

[6] L. Carlitz and John H. Hodges, Distribution of bordered symmetric, skew and hermitian matrices in a finite field, J. Reine Angew. Math. 195 (1955), 192-201 (1956). MR0075983 $(17,828 \mathrm{f})$

[7] John Cossey and Trevor Hawkes, Sets of p-powers as conjugacy class sizes, Proc. Amer. Math. Soc. 128 (2000), no. 1, 49-51, DOI 10.1090/S0002-9939-99-05138-2. MR.1641677 (2000c:20034)

[8] Anton Evseev, Reduction for characters of finite algebra groups, J. Algebra 325 (2011), 321-351, DOI 10.1016/j.jalgebra.2010.07.048. MR2745543(2012a:20024)

[9] Gustavo A. Fernández-Alcober and Alexander Moretó, On the number of conjugacy class sizes and character degrees in finite p-groups, Proc. Amer. Math. Soc. 129 (2001), no. 11, 3201-3204 (electronic), DOI 10.1090/S0002-9939-01-05946-9. MR.1844993 (2002d:20009)

[10] Jon González-Sánchez, Kirillov's orbit method for p-groups and pro-p groups, Comm. Algebra 37 (2009), no. 12, 4476-4488, DOI 10.1080/00927870802545679. MR2588861 (2011a:20057)

[11] P. Hall, The classification of prime-power groups, J. Reine Angew. Math. 182 (1940), 130141. MR0003389(2,211b)

[12] Marshall Hall Jr., The theory of groups, Chelsea Publishing Co., New York, 1976. Reprinting of the 1968 edition. MR0414669 (54 \#2765)

[13] Graham Higman, Enumerating p-groups. I. Inequalities, Proc. London Math. Soc. (3) 10 (1960), 24-30. MR0113948 (22 \#4779)

[14] Roger E. Howe, Kirillov theory for compact p-adic groups, Pacific J. Math. 73 (1977), no. 2, 365-381. MR0579176 (58 \#28314)

[15] Roger E. Howe, On representations of discrete, finitely generated, torsion-free, nilpotent groups, Pacific J. Math. 73 (1977), no. 2, 281-305. MR0499004 (58 \#16984)

[16] Bertram Huppert, A remark on the character-degrees of some p-groups, Arch. Math. (Basel) 59 (1992), no. 4, 313-318, DOI 10.1007/BF01197044. MR.1179454 (93g:20016)

[17] I. M. Isaacs, Sets of p-powers as irreducible character degrees, Proc. Amer. Math. Soc. 96 (1986), no. 4, 551-552, DOI 10.2307/2046302. MR826479(87d:20013)

[18] I. M. Isaacs, Counting characters of upper triangular groups, J. Algebra 315 (2007), no. 2, 698-719, DOI 10.1016/j.jalgebra.2007.01.027. MR2351888(2009a:20023)

[19] Noboru Ito and Avinoam Mann, Counting classes and characters of groups of prime exponent, Israel J. Math. 156 (2006), 205-220, DOI 10.1007/BF02773832. MR2282376 (2008i:20010)

[20] A. Jaikin-Zapirain, Zeta function of representations of compact p-adic analytic groups, J. Amer. Math. Soc. 19 (2006), no. 1, 91-118 (electronic), DOI 10.1090/S0894-0347-05-005011. MR2169043(2006f:20029)

[21] Marshall Hall Jr., A basis for free Lie rings and higher commutators in free groups, Proc. Amer. Math. Soc. 1 (1950), 575-581. MR0038336(12,388a)

[22] Thomas Michael Keller, Derived length and conjugacy class sizes, Adv. Math. 199 (2006), no. 1, 88-103, DOI 10.1016/j.aim.2004.11.002. MR2187399(2006k:20027)

[23] E. I. Khukhro, p-automorphisms of finite p-groups, London Mathematical Society Lecture Note Series, vol. 246, Cambridge University Press, Cambridge, 1998. MR1615819(99d:20029)

[24] Serge Lang and André Weil, Number of points of varieties in finite fields, Amer. J. Math. 76 (1954), 819-827. MR0065218 (16,398d) 
[25] Martin W. Liebeck and Aner Shalev, Character degrees and random walks in finite groups of Lie type, Proc. London Math. Soc. (3) 90 (2005), no. 1, 61-86, DOI 10.1112/S0024611504014935. MR2107038(2006h:20016)

[26] Christophe Reutenauer, Free Lie algebras, London Mathematical Society Monographs. New Series, vol. 7, The Clarendon Press Oxford University Press, New York, 1993. Oxford Science Publications. MR 1231799 (94j:17002)

[27] A. Stasinski and C. Voll, Representation zeta functions of nilpotent groups and generating functions for Weyl groups of type B, Amer. J. Math. 136 (2014), no. 2, 501-550, DOI 10.1353/ajm.2014.0010. MR3188068

[28] Ralph Stöhr and Michael Vaughan-Lee, Products of homogeneous subspaces in free Lie algebras, Internat. J. Algebra Comput. 19 (2009), no. 5, 699-703, DOI 10.1142/S0218196709005287. MR2547065 (2010i:17011)

[29] A. Vera López and J. M. Arregi, Conjugacy classes in Sylow p-subgroups of GL $(n, q)$. IV, Glasgow Math. J. 36 (1994), no. 1, 91-96, DOI 10.1017/S0017089500030597. MR.1260823 (94m:20052)

[30] Antonio Vera-López and J. M. Arregi, Conjugacy classes in unitriangular matrices, Linear Algebra Appl. 370 (2003), 85-124, DOI 10.1016/S0024-3795(03)00371-9. MR.1994321 (2004i:20091)

[31] Christopher Voll, Zeta functions of nilpotent groups-singular Pfaffians, Essays in geometric group theory, Ramanujan Math. Soc. Lect. Notes Ser., vol. 9, Ramanujan Math. Soc., Mysore, 2009, pp. 145-159. MR.2605359 (2011g:20049)

Department of Mathematics, University of Auckland, Auckland, New Zealand

E-mail address: obrien@math.auckland.ac.nz

School of Mathematics, University of Southampton, University Road, Southampton SO17 1BJ, United Kingdom

Current address: Fakultät für Mathematik, Universität Bielefeld, Postfach 100131, D-33501

Bielefeld, Germany

E-mail address: C.Voll.98@cantab.net 